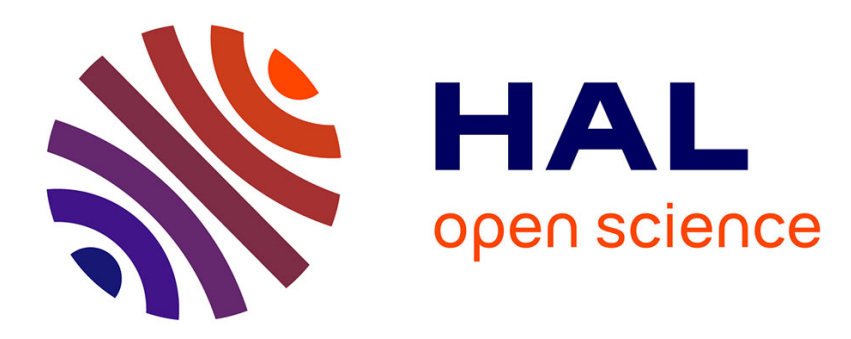

\title{
The Kelvin-Helmholtz instabilities in two-fluids shallow water models
}

\author{
David Lannes, Mei Ming
}

\section{To cite this version:}

David Lannes, Mei Ming. The Kelvin-Helmholtz instabilities in two-fluids shallow water models.

Fields Institute Communications, 2015, 75, pp.185-234. hal-01101993

\section{HAL Id: hal-01101993 \\ https://hal.science/hal-01101993}

Submitted on 14 Jan 2015

HAL is a multi-disciplinary open access archive for the deposit and dissemination of scientific research documents, whether they are published or not. The documents may come from teaching and research institutions in France or abroad, or from public or private research centers.
L'archive ouverte pluridisciplinaire HAL, est destinée au dépôt et à la diffusion de documents scientifiques de niveau recherche, publiés ou non, émanant des établissements d'enseignement et de recherche français ou étrangers, des laboratoires publics ou privés. 


\title{
The Kelvin-Helmholtz instabilities in two-fluids shallow water models
}

\author{
David Lannes and Mei Ming \\ To Walter Craig, for his 60th birthday, with friendship and admiration
}

\begin{abstract}
The goal of this paper is to describe the formation of Kelvin-Helmholtz instabilities at the interface of two fluids of different densities and the ability of various shallow water models to reproduce correctly the formation of these instabilities.

Working first in the so called rigid lid case, we derive by a simple linear analysis an explicit condition for the stability of the low frequency modes of the interface perturbation, an expression for the critical wave number above which KelvinHelmholtz instabilities appear, and a condition for the stability of all modes when surface tension is present. Similar conditions are derived for several shallow water asymptotic models and compared with the values obtained for the full Euler equations. Noting the inability of these models to reproduce correctly the scenario of formation of Kelvin-Helmholtz instabilities, we derive new models that provide a perfect matching. A comparisons with experimental data is also provided.

Moreover, we briefly discuss the more complex case where the rigid lid is replaced by a free surface. In this configuration, it appears that some frequency modes are stable when the velocity jump at the interface is large enough; we explain why such stable modes do not appear in the rigid lid case.
\end{abstract}

\section{Introduction}

Describing the motion of the surface of a non viscous fluid of constant density is known as the water waves problems and is now quite well-understood. The equations are known to be well-posed for smooth data under the Rayleigh-Taylor crite-

David Lannes

IMB, Université de Bordeaux et CNRS UMR 5251, 351 Cours de la Libération, 33405 Talence

Cedex, e-mail: David.Lannes@math.u-bordeaux1.fr

Mei Ming

School of Mathematics and Computational Science, Sun Yat-sen University, 135 Xingangxi Street, Guangzhou, 510275, China, e-mail: mingm@ mail.sysu.edu.cn 
rion stating that the vertical derivative of the pressure must be negative at the surface

$$
\text { (Rayleigh-Taylor criterion ) } \quad-\partial_{z} P_{\text {surf }}>0,
$$

which is equivalent to saying that the downward acceleration of the fluid must not exceed gravity; away from singularities, this condition is known to be always satisfied $[42,31]$. The solutions of the water waves equations are also known to be well-approximated by simpler asymptotic models in several asymptotic regimes. In particular, in the so called shallow water regime which is relevant for most applications to coastal oceanography or tsunami propagation for instance, many asymptotic models have been derived and justified, one of the pioneer works being Walter Craig's justification of the KdV approximation [17]. The so-called Nonlinear Shallow Water (or Saint-Venant) equations and the Green-Naghdi (or fully nonlinear Boussinesq, or Serre) equations are two examples of widely used models in the shallow water regime (in the sense that the depth is much smaller than the typical horizontal length); we refer to [33] for more details on these aspects.

The related problem consisting in describing the interface between two fluids of different density happens to be much more complicated due to the presence of a new kind of instabilities that do not exist in the water waves problem, the socalled Kelvin-Helmholtz instabilities, created by the discontinuity of the tangential velocity at the interface. If interfacial waves can be observed in spite of these instabilities, this is because some extra mechanism is involved which prevents the growth of Kelvin-Helmholtz instabilities. It has for instance been shown in [32] that a small amount of surface tension may be enough to control these instabilities, leading to the following generalization of the Rayleigh-Taylor criterion for the stability of two-fluids interfaces,

$$
-\llbracket \partial_{z} P_{\text {linterf }}^{ \pm} \rrbracket>\frac{1}{4} \frac{\left(\rho^{+} \rho^{-}\right)^{2}}{\sigma\left(\rho^{+}+\rho^{-}\right)^{2}} \mathfrak{c}(\zeta)\left|\llbracket \mathbf{U}_{\text {linterf }}^{ \pm} \rrbracket\right|_{\infty}^{4},
$$

where $\rho^{ \pm}$denote the densities of both fluids, $\sigma$ the surface tension coefficient, $\mathfrak{c}(\zeta)$ a constant of little importance, and $\llbracket \mathbf{U}_{\text {linterf }}^{ \pm} \rrbracket$ the velocity jump at the interface. This criterion is the result of two mechanisms: gravity stabilizes the low frequency components of the interfacial waves, while surface tension stabilizes the high frequency modes. The stability of the low frequency modes is not always granted: the relative gravity $g^{\prime}=g \frac{\rho^{+}-\rho^{-}}{\rho^{+}+\rho^{-}}$must be large enough. From the analysis of [32] this can be expressed as follows,

$$
g^{\prime}>\frac{\rho^{+} \rho^{-}}{\left(\rho^{+}+\rho^{-}\right)^{2}} C(\zeta)\left|\llbracket \mathbf{U}_{\text {linterf }}^{ \pm} \rrbracket\right|_{\infty}^{2},
$$

for some constant $C(\zeta)$ depending on $\zeta$ (and homogeneous to the inverse of a length).

If this condition is satisfied, then there is a critical wave number $k_{K H}$ such that Kelvin-Helmholtz instabilities appear above this wave number in the absence of surface tension. If $k_{K H}$ is large enough, then we may expect a regularization by 
small scale processes: capillarity (as in [32]), viscosity, etc. A precise description of $k_{K H}$ is therefore important.

A precise evaluation of $k_{K H}$ in the general nonlinear case seems out of reach because the computations based on the linearization around any solution is extremely technical. It is however possible to get some interesting insight on the formation of Kelvin-Helmholtz instabilities by considering the linearization around a constant shear, and this is the approach we shall use in this paper. For instance, we shall show that a necessary and sufficient condition for the stability of the low frequency modes for the linearized system around a shear flow with constant speeds $c^{+}$and $c^{-}$is

$$
\left|\llbracket c^{ \pm} \rrbracket\right|^{2}<\Omega_{K H}:=\frac{g^{\prime} H_{0}}{\underline{\rho}^{+} \underline{\rho}^{-}}, \quad \text { with } H_{0}=\underline{\rho}^{+} H^{-}+\underline{\rho}^{-} H^{+}
$$

(with $\underline{\rho}^{ \pm}=\frac{\rho^{ \pm}}{\rho^{+}+\rho^{-}}$denoting the relative densities of the fluids), a condition which is both consistent with (2), and more explicit. Moreover, the technical simplifications brought by linearizing around a constant state allow us to give a formula for $k_{K H}$ when the above condition is satisfied. We are also able to derive a condition for the stability of all modes, which is therefore a linear but more precise version of (1),

$$
\llbracket c^{ \pm} \rrbracket^{2} \leq \Omega^{c r} \quad \text { with } \quad \Omega^{c r} \sim \frac{2}{\sqrt{\mathrm{Bo}}} \Omega_{K H}
$$

and where Bo is the Bond number

$$
\mathrm{Bo}=\frac{\left(\rho^{+}+\rho^{-}\right) g^{\prime} H_{0}^{2}}{\sigma} .
$$

Since the Bond number is very large in most cases, this condition is much more restrictive than (3).

As for the one fluid (water waves) case, simpler asymptotic models are often used to describe the propagation of interfacial waves. We will stick here to the shallow water regime (in which the depth of both fluids are small compared to the typical horizontal length); we refer to [8] for a systematic derivation of asymptotic models in this context (see also [25] for a spectacular application of such models for the explanation of the dead water phenomenon).

Since shallow water models somehow only keep the low frequency part of the wave, they behave differently with respect to Kelvin-Helmholtz instabilities and may be well posed without stabilizing phenomenons such as surface tension. Their well-posedness is however subject to additional conditions on the data, and it turns out that these conditions are actually what remains of the Kelvin-Helmholtz instabilities for these models. The singularity corresponding to the violation of these extra conditions must therefore be interpreted as the prediction of a Kelvin-Helmholtz instability. One of the questions we raise here is: how well do the various shallow water models approximate the scenario of formation of Kelvin-Helmholtz instabilities of the full Euler equations? This is to our knowledge the first time this issue is addressed. 
Our strategy to answer this question is to consider the same problem as for the full Euler equations and that we described above. Namely, we consider the stability of the linearization of the various shallow water models around a constant shear. We exhibit a condition for the low frequency stability that we compare to (3), and a critical wave number $k_{K H}^{\text {app }}$ for the apparition of the Kelvin-Helmholtz instabilities, and that we compare to $k_{K H}$. We also compute a condition for the stability of all modes of the form $\llbracket c^{ \pm} \rrbracket^{2} \leq \Omega^{c r, a p p}$ that we compare to (4).

The asymptotic models we consider here are the so-called Shallow Water/Shallow Water (SW/SW) model which is the model obtained at first order in the shallow water limit. We show that this model underestimates the Kelvin-Helmholtz instabilities. We then consider the Green-Naghdi/Green-Naghdi model which is obtained in the same regime as the SW/SW model, but which is precise up to second order. This model overestimates the Kelvin-Helmholtz instabilities. Moreover, we derive two new families of regularized GN/GN model (one of which being a generalization of the models derived in [13]), and we show that they are able to reproduce exactly the singularity formation scenario of the full Euler equations. These results are summed up in the following table, and used to interpret some experimental measurements (see $\S 4.3)$.

Table 1 Stability criteria for Euler system and different models

\begin{tabular}{llll}
\hline & LF stability & Critical wave number & Stability of all modes \\
\hline Euler & $\llbracket c^{ \pm} \rrbracket<\Omega_{K H}$ & $0<k_{K H}<\infty$ & $\Omega^{c r} \sim \frac{2}{\sqrt{\mathrm{Bo}}} \Omega_{K H}<\Omega_{K H}$ \\
SW/SW & idem & $k_{S W}=\infty$ & $\Omega_{S W}^{c r}=\Omega_{K H}>\Omega^{c r}$ \\
GN/GN & idem & $k_{G N, \sigma}<k_{K H}$ & $\Omega_{G N_{\sigma}}^{c r}<\Omega^{c r}$ \\
GN/GN & idem & $k_{G N_{r}}=k_{K H}$ possible & $\Omega_{G N_{r}}^{c r}=\Omega^{c r}$ possible \\
\hline
\end{tabular}

All the results described above are obtained in the framework of the rigid lid approximation for which the fluid above is bounded from above by a rigid bottom. We also consider here the free surface case where the upper boundary is now, like the interface, a free surface. This framework is of course relevant for many applications and has been considered for instance in $[19,3,20,21,1,24,26,11,40]$. We follow the same approach as above: we exhibit a condition for the stability of low frequency modes, and a stronger condition for the stability of all modes. The problem is however much more technical than for the rigid lid case, and we resort to numerical computations. As noticed in $[35,1,11,40]$, the free surface case is also marked by a peculiar phenomenon: low frequency modes are stable for small shears as in the rigid lid case, but also for large enough shears.

As in the rigid lid case, we compare the formation of Kelvin-Helmholtz instabilities for the full Euler equations and for a Shallow Water/Shallow Water model with free surface. Except for a special configuration (the depth of both fluids is the same) where we can carry the computations out, this comparison is numerical. The investigation of the next order models $(\mathrm{GN} / \mathrm{GN})$ is not done in this paper because it is highly technical, and because it can be done following the same approach as in the 
rigid lid case. Note that GN/GN type models have been studied in the free surface case in [1], and that it is possible to generalize this study by deriving regularized models with the techniques used here in the rigid lid case in order to obtain a better description of the formation of Kelvin-Helmholtz instabilities.

The last point we address in this paper is a study of the behavior of the additional stability area observed for large shears in the free surface case. More precisely, we want to understand how this stability area behaves in the so called rigid lid limit. We infer from this study that the rigid lid approximation can only be true for very small density contrast between both fluids, and that the large shear stability area disappears in the rigid lid case because it corresponds only to infinite shears.

\subsection{Organization of the paper}

We first recall in Section 2 the equations of motions, both in the rigid lid and in the free surface cases. We also derive the reduced formulations that are used throughout the paper for the computations. The Kelvin-Helmholtz instabilities are then studied in Section 3 for the full equations in the rigid lid case. In Section 4, we study several shallow water models, focusing our attention on their ability to describe the KelvinHelmholtz instabilities. An experimental application is also given. We then turn to study, in Section 5, the formation of Kelvin-Helmholtz instabilities for the full Euler equations in the free surface case. The shallow water behavior of these instabilities and the behavior of the large shear stability area is then investigated in Section 6.

\subsection{Notations}

- If $A^{+}$and $A^{-}$are two quantities (real numbers, functions, etc.), the notations $\llbracket A^{ \pm} \rrbracket$ and $\left\langle A^{ \pm}\right\rangle$stand for

$$
\llbracket A^{ \pm} \rrbracket=A^{+}-A^{-} \quad \text { and } \quad\left\langle A^{ \pm}\right\rangle=\frac{A^{+}+A^{-}}{2} .
$$

- We use relative densities

$$
\underline{\rho}^{+}=\frac{\rho^{+}}{\rho^{+}+\rho^{-}}, \quad \underline{\rho}^{-}=\frac{\rho^{-}}{\rho^{+}+\rho^{-}}
$$

as the ratios of densities $\rho^{+}, \rho^{-}$for the lower and upper layers respectively.

- Parameters $\delta$ and $\gamma$ stand for the depth ratio $\delta=H^{-} / H^{+}$and the density ratio $\gamma=\underline{\rho}^{-} / \underline{\rho}^{+}$respectively.

- The notations $\mathrm{th}^{ \pm}, \mathrm{cth}^{ \pm}, \mathrm{sh}^{ \pm}$and $\mathrm{ch}^{ \pm}$stand for the hyperbolic Fourier multipliers $\tanh \left(H^{ \pm}|D|\right), \operatorname{coth}\left(H^{ \pm}|D|\right), \sinh \left(H^{ \pm}|D|\right)$ and $\cosh \left(H^{ \pm}|D|\right)$, with $i D=\nabla$ and $H^{ \pm}$the average depths for the upper and lower fluids. When no misunder- 
standing is possible, we also take $\mathrm{th}^{ \pm}, \mathrm{cth}^{ \pm}, \mathrm{sh}^{ \pm}$and $\mathrm{ch}^{ \pm}$as their Fourier modes $\tanh \left(H^{ \pm}|\mathbf{k}|\right), \operatorname{coth}\left(H^{ \pm}|\mathbf{k}|\right), \sinh \left(H^{ \pm}|\mathbf{k}|\right)$ and $\cosh \left(H^{ \pm}|\mathbf{k}|\right)$.

\section{The equations of motion}

In this section, we introduce briefly the equations of motion for both the rigid lid case and the free surface case.

\subsection{The rigid lid case}

We consider first the case of a rigid lid; in such a configuration (see Figure 1), the inferior fluid domain $\Omega_{t}^{+}$is delimited from below by a flat bottom $\Gamma^{+}$at height $z=-H^{+}$and from above by the interface $\Gamma_{t}=\left\{(X, z) \in \mathbb{R}^{d} \times \mathbb{R}, z=\zeta(t, X)\right\}$, and the superior fluid is bounded from below by $\Gamma_{t}$ and from above by a rigid lid $\Gamma^{-}$at height $z=H^{-}$.

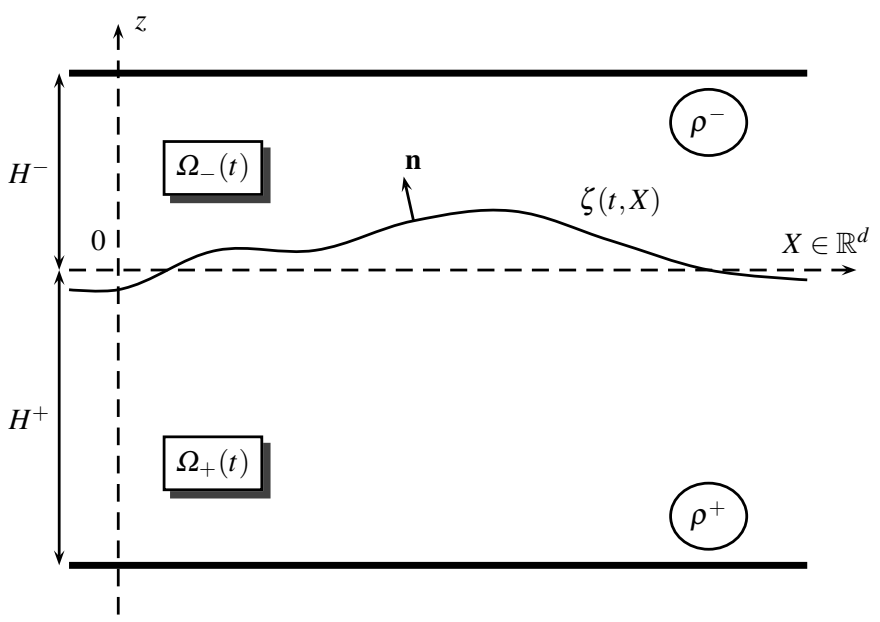

Fig. 1 The rigid lid configuration

\subsubsection{Basic equations}

We denote by $\mathbf{U}^{ \pm}$the velocity field in $\Omega_{t}^{ \pm}$; the horizontal component of $\mathbf{U}^{ \pm}$is $V^{ \pm}$ and its vertical one $w^{ \pm}$. The pressure is denoted by $P^{ \pm}$. The equations of motion are then the following, 
- Equations in the fluid layers. In both fluid layers, the velocity field $\mathbf{U}^{ \pm}$and the pressure $P^{ \pm}$satisfy the equations

$$
\operatorname{div} \mathbf{U}^{ \pm}(t, \cdot)=0, \quad \operatorname{curl} \mathbf{U}^{ \pm}(t, \cdot)=0, \quad \text { in } \Omega_{t}^{ \pm} \quad(t \geq 0),
$$

which express the incompressibility and irrotationality assumptions, and

$$
\rho^{ \pm}\left(\partial_{t} \mathbf{U}^{ \pm}+\left(\mathbf{U}^{ \pm} \cdot \nabla_{X, z}\right) \mathbf{U}^{ \pm}\right)=-\nabla_{X, z} P^{ \pm}-g \mathbf{e}_{z} \quad \text { in } \Omega_{t}^{ \pm} \quad(t \geq 0)
$$

which expresses the conservation of momentum (Euler equation).

- Boundary conditions at the rigid bottom and lid. Impermeability of these two boundaries is classically rendered by

$$
\left.w^{+}(t, \cdot)\right|_{\Gamma^{+}}=0, \text { and }\left.w^{-}(t, \cdot)\right|_{\Gamma^{-}}=0 \quad(t \geq 0) .
$$

- Boundary conditions at the moving interface. The fact that the interface is a bounding surface (the fluid particles do not cross it) yields the equations

$$
\partial_{t} \zeta-\sqrt{1+|\nabla \zeta|^{2}} \mathbf{U}_{n}^{ \pm}=0, \quad(t \geq 0)
$$

where $\mathbf{U}_{n}^{ \pm}:=\mathbf{U}^{ \pm}{ }_{\Gamma_{t}} \cdot \mathbf{n}, \mathbf{n}$ being the upward unit normal vector to the interface $\Gamma_{t}$. A direct consequence of (9) is that there is no jump of the normal component of the velocity at the interface. Finally, the continuity of the stress tensor at the interface gives

$$
\llbracket P^{ \pm}(t, \cdot)_{\Gamma_{t}} \rrbracket=\sigma \kappa(\zeta), \quad(t \geq 0),
$$

where $\sigma \geq 0$ is the surface tension coefficient and

$$
\kappa(\zeta)=-\nabla \cdot\left(\frac{\nabla \zeta}{\sqrt{1+|\nabla \zeta|^{2}}}\right)
$$

is the mean curvature of the interface.

\subsubsection{Reduction to the interface}

As stressed above, the normal component of the velocity field $\mathbf{U}^{ \pm} \cdot \mathbf{n}$ is continuous at the interface. This is not the case for the tangential component of the velocity. Since the vertical component of this vector can be deduced from its horizontal components, we will focus our attention on these horizontal components and more specifically on the quantity $U_{\|}^{ \pm}$defined as

$$
\mathbf{U}_{\left.\right|_{z=\zeta} ^{ \pm}}^{ \pm} \times \mathbf{N}=\left(\begin{array}{c}
-\left(U_{\|}^{ \pm}\right)^{\perp} \\
-\left(U_{\|}^{ \pm}\right)^{\perp} \cdot \nabla \zeta
\end{array}\right), \quad \text { with } \quad \mathbf{N}=\sqrt{1+|\nabla \zeta|^{2}} \mathbf{n}
$$


Taking the vector product of the trace of (7) at the interface with $\mathbf{N}$, and considering only the horizontal components of the resulting equation, one readily gets

$$
\partial_{t} U_{\|}^{ \pm}+g \nabla \zeta+\frac{1}{2} \nabla\left|U_{\|}^{ \pm}\right|^{2}-\frac{1}{2} \nabla\left(\left(1+|\nabla \zeta|^{2}\right)\left|\underline{w}^{ \pm}\right|^{2}\right)=-\frac{1}{\rho^{ \pm}} \nabla \underline{P}^{ \pm},
$$

where we denoted

$$
\underline{P}^{ \pm}:=P_{\left.\right|_{z=\zeta} ^{ \pm}}^{ \pm}, \quad \underline{\mathbf{U}}^{ \pm}=\left(\underline{V}^{ \pm}, \underline{w}^{ \pm}\right):=\mathbf{U}_{\left.\right|_{z=\zeta} ^{ \pm}}^{ \pm}=\left(V_{\left.\right|_{z=\zeta} ^{ \pm}}^{ \pm}, w_{\left.\right|_{z=\zeta}}^{ \pm}\right)
$$

The interfacial waves equations (6)-(10) can therefore be recast as two sets of two equations on the fixed domain $\mathbb{R}_{t}^{+} \times \mathbb{R}_{X}^{d}$, i.e. on the interface

$$
\left\{\begin{array}{l}
\partial_{t} \zeta-\underline{\mathbf{U}}^{ \pm} \cdot \mathbf{N}=0 \\
\partial_{t} U_{\|}^{ \pm}+g \nabla \zeta+\frac{1}{2} \nabla\left|U_{\|}^{ \pm}\right|^{2}-\frac{1}{2} \nabla\left(\left(1+|\nabla \zeta|^{2}\right)\left|\underline{w}^{ \pm}\right|^{2}\right)=-\frac{1}{\rho^{ \pm}} \nabla \underline{P}^{ \pm}
\end{array}\right.
$$

with the jump condition

$$
\llbracket P^{ \pm}(t, \cdot)_{\Gamma_{t}} \rrbracket=-\sigma \nabla \cdot\left(\frac{\nabla \zeta}{\sqrt{1+|\nabla \zeta|^{2}}}\right) .
$$

Remark 1. i- In (11), the incompressibility and irrotationality conditions (6) imply ${ }^{1}$ that one can express $\underline{\mathbf{U}}^{ \pm} \cdot \mathbf{N}$ in terms of $\zeta$ and the tangential component $U_{\|}^{ \pm}$.

ii- It is possible to reduce further (11) to one set of two equations. Let us define

$$
U_{\|}=\underline{\rho}^{+} U_{\|}^{+}-\underline{\rho}^{-} U_{\|}^{-}
$$

multiplying the second equation of $(11)_{ \pm}$by $\underline{\rho}^{+}$and subtracting the resulting + and - equations, we obtain

$$
\left\{\begin{aligned}
\partial_{t} \zeta-\underline{\mathbf{U}}^{ \pm} \cdot \mathbf{N}=0 \\
\partial_{t} U_{\|}+g^{\prime} \nabla \zeta+\frac{1}{2} \nabla \llbracket \underline{\rho}^{ \pm}\left|U_{\|}^{ \pm}\right|^{2} \rrbracket-\frac{1}{2} \nabla\left(\left(1+|\nabla \zeta|^{2}\right) \llbracket \underline{\rho}^{ \pm}\left|\underline{w}^{ \pm}\right|^{2} \rrbracket\right) \\
=-\frac{\sigma}{\rho^{+}+\rho^{-}} \nabla \kappa(\zeta)
\end{aligned}\right.
$$

where $g^{\prime}=g\left(\underline{\rho}^{+}-\underline{\rho}^{-}\right)$is the reduced gravity. It is possible to show that $\underline{\mathbf{U}}^{+} \cdot \mathbf{N}=$ $\underline{\mathbf{U}}^{-} \cdot \mathbf{N}, U_{\|}^{ \pm}$and $\underline{w}^{ \pm}$can be expressed in terms of $\zeta$ and $U_{\|}$, so that (13) is a closed set of equations on $\zeta$ and $U_{\|}$.

iii- From the incompressibility and irrotationality assumptions (6), we infer that the velocity fields $\mathbf{U}^{ \pm}$derive from a scalar harmonic potential $\Phi^{ \pm}$(i.e. $\mathbf{U}^{ \pm}=$ $\nabla_{X, z} \Phi^{ \pm}$, with $\Delta_{X, z} \Phi^{ \pm}=0$ in $\left.\Omega_{t}^{ \pm}\right)$. Denoting by $\psi^{ \pm}$the traces of $\Phi^{ \pm}$at the

\footnotetext{
${ }^{1}$ It has been proved recently [12] in the particular case of one single fluid $\left(\rho^{-}=0\right)$ that the irrotationality condition is not needed and that (11), together with the standard evolution equation for the vorticity, form a closed, well-posed, set of equations.
} 
interface, $\psi^{ \pm}=\Phi_{\left.\right|_{z=\zeta} ^{ \pm}}^{ \pm}$, one can check that $U_{\|}^{ \pm}=\nabla \psi^{ \pm}$. Introducing

$$
\psi=\underline{\rho}^{+} \psi^{+}-\underline{\rho}^{-} \psi^{-},
$$

one has $U_{\|}=\nabla \psi$ and the second equation in (13) can be replaced by a scalar equation on $\psi$, leading to

$$
\left\{\begin{array}{l}
\partial_{t} \zeta-\sqrt{1+|\nabla \zeta|^{2}} \mathbf{U}_{n}^{ \pm}=0, \\
\partial_{t} \psi+g^{\prime} \zeta+\frac{1}{2} \llbracket \underline{\rho}^{ \pm}\left|U_{\|}^{ \pm}\right|^{2} \rrbracket-\frac{1}{2}\left(1+|\nabla \zeta|^{2}\right) \llbracket \underline{\rho}^{ \pm}\left|\underline{w}^{ \pm}\right|^{2} \rrbracket=-\frac{\sigma}{\rho^{+}+\rho^{-}} \kappa(\zeta) .
\end{array}\right.
$$

This is the formulation used in [32] to establish the nonlinear well posedness of the two-fluids interfaces equations. In the case of one fluid (water waves), these equations coincide with the Zakharov-Craig-Sulem formulation of the water waves equations [43, 22]. One of the main features of this formulation is its Hamiltonian structure; it is remarkable that the two-fluid generalization of the Zakharov-Craig-Sulem formulation is also Hamiltonian, as remarked in [4] (see in particular $\S 5$ of that reference for considerations on Kelvin-Helmholtz instabilities). Using asymptotic expansions of the Hamiltonian, Craig, Guyenne and Kalisch derived in [19] a family of Hamiltonian approximations to the two-fluids equations (14).

\subsection{The free surface case}

We consider here the case of a free surface (see Figure 2). The configuration is the same as in $\S 2.1$ except that the superior fluid is now bounded from above by a free surface $\Gamma_{t}^{s}=\left\{(X, z) \in \mathbb{R}^{d} \times \mathbb{R}, z=\zeta^{s}(t, X)\right\}$.

\subsubsection{Basic equations}

The only difference between the free surface and rigid lid cases is that the boundary condition (8) must be replaced by the kinematic condition

$$
\partial_{t} \zeta^{s}-\sqrt{1+\left|\nabla \zeta^{s}\right|} \mathbf{U}_{n^{s}}^{-}=0,
$$

where $\mathbf{U}_{n^{s}}^{-}:=\mathbf{U}_{\left.\right|_{t} ^{s}}^{-} \cdot \mathbf{n}^{s}, \mathbf{n}^{s}$ being the upward unit normal vector to the free surface $\Gamma_{t}^{s}$, and that the value of the pressure at the free surface must be prescribed; neglecting the effects of surface tension at the surface, the pressure is constant, and since it is defined up to a constant, we take it equal to zero,

$$
P_{p_{z=\zeta^{s}}}=0 .
$$




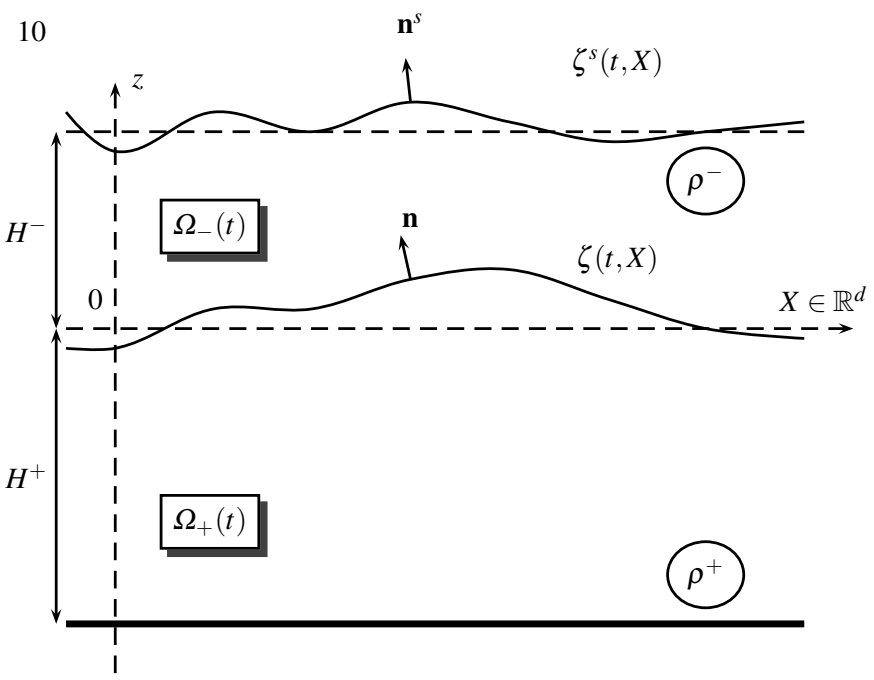

D. Lannes and M. Ming

Fig. 2 The free surface configuration

\subsubsection{Reduction to the interface}

Consistently with the notations previously used, we define the quantity $U_{\|}^{s}$ as

$$
\mathbf{U}_{\left.\right|_{z=\zeta^{s}}}^{-} \times \mathbf{N}^{s}=\left(\begin{array}{c}
-\left(U_{\|}^{s}\right)^{\perp} \\
-\left(U_{\|}^{s}\right)^{\perp} \cdot \nabla \zeta^{s}
\end{array}\right), \quad \text { with } \quad \mathbf{N}^{s}=\sqrt{1+\left|\nabla \zeta^{s}\right|^{2}} \mathbf{n}^{s} .
$$

Taking the vector product of the trace of (7) at the free surface with $\mathbf{N}^{s}$, and considering only the horizontal components of the resulting equation, one readily gets

$$
\partial_{t} U_{\|}^{s}+g \nabla \zeta^{s}+\frac{1}{2} \nabla\left|U_{\|}^{s}\right|^{2}-\frac{1}{2} \nabla\left(\left(1+\left|\nabla \zeta^{s}\right|^{2}\right)\left|\underline{w}^{s}\right|^{2}\right)=0
$$

where we denoted

$$
\underline{\mathbf{U}}^{s}=\left(\underline{V}^{s}, \underline{w}^{s}\right):=\mathbf{U}_{\left.\right|_{z=\zeta^{s}} ^{-}}=\left(V_{\left.\right|_{z=\zeta^{s}} ^{-}}^{-}, w_{\left.\right|_{z=\zeta^{s}}}^{-}\right)
$$

and the surface tension at the top free surface is neglected. The interfacial waves equations (6)-(10) can therefore be recast as three sets of two equations on the fixed domain $\mathbb{R}_{t}^{+} \times \mathbb{R}_{X}^{d}$, namely,

$$
\left\{\begin{array}{l}
\partial_{t} \zeta^{s}-\underline{\mathbf{U}}^{s} \cdot \mathbf{N}^{s}=0, \\
\partial_{t} U_{\|}^{s}+g \nabla \zeta^{s}+\frac{1}{2} \nabla\left|U_{\|}^{s}\right|^{2}-\frac{1}{2} \nabla\left(\left(1+\left|\nabla \zeta^{s}\right|^{2}\right)\left|\underline{w}^{s}\right|^{2}\right)=0,
\end{array}\right.
$$

and 


$$
\left\{\begin{array}{l}
\partial_{t} \zeta-\underline{\mathbf{U}}^{ \pm} \cdot \mathbf{N}=0 \\
\partial_{t} U_{\|}^{ \pm}+g \nabla \zeta+\frac{1}{2} \nabla\left|U_{\|}^{ \pm}\right|^{2}-\frac{1}{2} \nabla\left(\left(1+|\nabla \zeta|^{2}\right)\left|\underline{w}^{ \pm}\right|^{2}\right)=-\frac{1}{\rho^{ \pm}} \nabla \underline{P}^{ \pm}
\end{array}\right.
$$

with the jump condition

$$
\llbracket P^{ \pm}(t, \cdot)_{\left.\right|_{t}} \rrbracket=\sigma \kappa(\zeta) .
$$

Remark 2. Proceeding as in Remark 1, it is possible to reduce this set of seven equations into a set of four equations on $\left(\zeta^{s}, U_{\|}^{s}, \zeta, U_{\|}\right)$, with $U_{\|}=\underline{\rho}^{+} U_{\|}^{+}-\underline{\rho}^{-} U_{\|}^{-}$These equations are

$$
\left\{\begin{array}{l}
\partial_{t} \zeta^{s}-\underline{\mathbf{U}}^{s} \cdot \mathbf{N}^{s}=0 \\
\partial_{t} U_{\|}^{s}+g \nabla \zeta^{s}+\frac{1}{2} \nabla\left|U_{\|}^{s}\right|^{2}-\frac{1}{2} \nabla\left(\left(1+\left|\nabla \zeta^{s}\right|^{2}\right)\left|\underline{w}^{s}\right|^{2}\right)=0 \\
\partial_{t} \zeta-\underline{\mathbf{U}}^{ \pm} \cdot \mathbf{N}=0 \\
\partial_{t} U_{\|}+g^{\prime} \nabla \zeta+\frac{1}{2} \nabla \llbracket \underline{\rho}^{ \pm}\left|U_{\|}^{ \pm}\right|^{2} \rrbracket-\frac{1}{2} \nabla\left(\left(1+|\nabla \zeta|^{2}\right) \llbracket \underline{\rho}^{ \pm}\left|\underline{w}^{ \pm}\right|^{2} \rrbracket\right) \\
=-\frac{\sigma}{\rho^{+}+\rho^{-}} \nabla \kappa(\zeta)
\end{array}\right.
$$

where $g^{\prime}=g\left(\rho^{+}-\rho^{-}\right)$is the reduced gravity. Giving a version of these equations in terms of velocity potential similar to (14) in the rigid lid case is of course possible.

\section{The Kelvin-Helmholtz instability in the rigid lid case}

The formation of Kelvin-Helmholtz instabilities for two-fluids interfaces and in the case of a rigid lid is investigated here. In this setting, it is known [32] that the full nonlinear equations are well posed under the generalized Rayleigh-Taylor criterion (1). This criterion is a sufficient condition ensuring the stability of all frequency modes. By sticking to the much simpler linear theory, we want to get additional information that will also be used to compare with the formation of Kelvin-Helmholtz instabilities in shallow water asymptotic models and in the free surface case, where the nonlinear analysis is still an open problem. More precisely, we consider the linear stability of perturbations to a constant shear flow. We insist on three important aspects: 1) the existence of a critical value of the shear flow below which low frequency modes are stable 2) the existence for such flows of a critical wavenumber delimiting this stability region, and 3) the possible existence of a second critical shear flow below which all modes are stable. These stability criteria are studied in detail, and explicit formulas or accurate approximations are provided, which allows one to comment on the influence of the density ratio $\gamma=\rho^{-} / \rho^{+}$and of the depth ratio $\delta=H^{-} / H^{+}$for instance. 


\subsection{Linearization around a constant shear $\left(\mathrm{c}^{+}, \mathrm{c}^{-}\right)$}

We consider here the linear equations governing small perturbations of the constant horizontal shear $\zeta=0, V^{ \pm}=\mathbf{c}^{ \pm}, w^{ \pm}=0$ (and therefore $U_{\|}^{ \pm}=\mathbf{c}^{ \pm}$).

Linearizing (10)-(11) around $\zeta=0, V^{ \pm}=\mathbf{c}^{ \pm}$and $w^{ \pm}=0$ we find the following linear equations for the perturbation $\left(\dot{\zeta}, \dot{V}^{ \pm}, \dot{w}^{ \pm}\right)$

$$
\left\{\begin{array}{l}
\partial_{t} \dot{\zeta}+\mathbf{c}^{ \pm} \cdot \nabla \dot{\zeta}-\dot{w}^{ \pm}=0 \\
\partial_{t} \dot{V}^{ \pm}+g \nabla \dot{\zeta}+\mathbf{c}^{ \pm} \cdot \nabla \dot{V}^{ \pm}=-\frac{1}{\rho^{ \pm}} \nabla \underline{P}^{ \pm}
\end{array}\right.
$$

with

$$
\llbracket \underline{P}^{ \pm} \rrbracket=-\sigma \Delta \dot{\zeta}
$$

In the same spirit as in Remark 1, we can derive a reduced formulation for these equations; denoting $\dot{V}=\underline{\rho}^{+} \dot{V}^{+}-\underline{\rho}^{-} \dot{V}^{-}$and remarking that

$$
\llbracket \underline{\rho}^{ \pm} \mathbf{c}^{ \pm} \cdot \dot{V}^{ \pm} \rrbracket=\left\langle\mathbf{c}^{ \pm}\right\rangle \cdot \dot{V}+\llbracket \mathbf{c}^{ \pm} \rrbracket \cdot\left\langle\underline{\rho}^{ \pm} \dot{V}^{ \pm}\right\rangle,
$$

we first get

$$
\left\{\begin{array}{l}
\partial_{t} \dot{\zeta}+\mathbf{c}^{ \pm} \cdot \nabla \dot{\zeta}-\dot{w}^{ \pm}=0 \\
\partial_{t} \dot{V}+\left\langle\mathbf{c}^{ \pm}\right\rangle \cdot \nabla \dot{V}+g^{\prime} \nabla \dot{\zeta}+\llbracket \mathbf{c}^{ \pm} \rrbracket \cdot \nabla\left\langle\underline{\rho}^{ \pm} \dot{V}^{ \pm}\right\rangle=\frac{\sigma}{\rho^{+}+\rho^{-}} \nabla \Delta \dot{\zeta}
\end{array}\right.
$$

In order to prove that these equations are in closed form, we still need to express $\dot{V}^{ \pm}$and $\dot{w}^{ \pm}$in terms of $\dot{\zeta}$ and $\dot{V}$. This is done in the following lemma.

Lemma 1. One has

$$
\begin{aligned}
& \dot{V}^{+}=\frac{\underline{\rho}^{-}}{\underline{\rho}^{-} \mathrm{th}^{+}+\underline{\rho}^{+} \mathrm{th}^{-}} \frac{\llbracket \mathbf{c}^{ \pm} \rrbracket \cdot \nabla}{|D|} \nabla \dot{\zeta}+\frac{\mathrm{th}^{-}}{\underline{\rho}^{-} \mathrm{th}^{+}+\underline{\rho}^{+} \mathrm{th}^{-}} \dot{V} \\
& \dot{V}^{-}=\frac{\underline{\rho}^{+}}{\underline{\rho}^{-} \mathrm{th}^{+}+\underline{\rho}^{+} \mathrm{th}^{-}} \frac{\llbracket \mathbf{c}^{ \pm} \rrbracket \cdot \nabla}{|D|} \nabla \dot{\zeta}-\frac{\mathrm{th}^{+}}{\underline{\rho}^{-} \mathrm{th}^{+}+\underline{\rho}^{+} \mathrm{th}^{-}} \dot{V}
\end{aligned}
$$

and $\dot{w}^{ \pm}=\mp \operatorname{th}^{ \pm} \frac{\nabla}{|D|} \cdot \dot{V}^{ \pm}$.

Proof. From the first equation of (21), we get

$$
\dot{w}^{+}-\dot{w}^{-}=\llbracket \mathbf{c}^{ \pm} \rrbracket \cdot \nabla \dot{\zeta}
$$

(this is the linear version of the continuity of the normal component of the velocity). For the same reason as in the third point of Remark 1, we can also write $\dot{V}^{ \pm}=\nabla \dot{\psi}^{ \pm}$ and $\dot{V}=\nabla \dot{\psi}$, with $\dot{\psi}=\underline{\rho}^{+} \dot{\psi}^{+}-\underline{\rho}^{-} \dot{\psi}^{-}$, and where

$$
\dot{\psi}^{ \pm}=\Phi_{\left.\right|_{z=0} ^{ \pm}}^{ \pm} \quad \text { with } \quad \Delta_{X, z} \Phi^{ \pm}=0 \quad \text { in }-H^{ \pm}< \pm z<0 \quad \text { and } \partial_{z} \Phi_{\left.\right|_{z=\mp H^{ \pm}} ^{ \pm}}=0 .
$$


From a simple linear analysis, we get therefore

$$
\dot{w}^{ \pm}= \pm|D| \operatorname{th}^{ \pm} \dot{\psi}^{ \pm}
$$

Since moreover, one has by definition $\dot{\psi}=\underline{\rho}^{+} \dot{\psi}^{+}-\underline{\rho}^{-} \dot{\psi}^{-}$, one can express $\dot{\psi}^{ \pm}$ (and therefore $\dot{V}^{ \pm}=\nabla \dot{\psi}^{ \pm}$and $\dot{w}^{ \pm}$through the above relation) in terms of $\dot{V}=\nabla \dot{\psi}$ and $\dot{\zeta}$ by solving the system

$$
\begin{cases}|D| \mathrm{th}^{+} \dot{\psi}^{+}+|D| \mathrm{th}^{-} \dot{\psi}^{-} & =\llbracket \mathbf{c}^{ \pm} \rrbracket \cdot \nabla \dot{\zeta} \\ \underline{\rho}^{+} \dot{\psi}^{+}-\underline{\rho}^{-} \dot{\psi}^{-} & =\dot{\psi}\end{cases}
$$

which leads to the formula of the lemma.

Thanks to the lemma, we can rewrite (21) under the form

$$
\left\{\begin{array}{l}
\partial_{t} \dot{\zeta}+\mathbf{c}(D) \cdot \nabla \dot{\zeta}+\frac{\mathrm{th}^{+} \mathrm{th}^{-}}{\rho^{-} \mathrm{th}^{+}+\underline{\rho}^{+} \mathrm{th}^{-}} \frac{\nabla}{|D|} \cdot \dot{V}=0 \\
\partial_{t} \dot{V}+\mathbf{c}(D) \cdot \nabla \dot{V}+\left(g^{\prime}-e(D)-\frac{\sigma}{\rho^{+}+\rho^{-}} \Delta\right) \nabla \dot{\zeta}=0
\end{array}\right.
$$

with

$$
\mathbf{c}(D)=\frac{\mathbf{c}^{+} \underline{\rho}^{+} \mathrm{th}^{-}+\mathbf{c}^{-} \underline{\rho}^{-} \mathrm{th}^{+}}{\underline{\rho}^{-} \mathrm{th}^{+}+\underline{\rho}^{+} \mathrm{th}^{-}}, \quad e(D)=\frac{\underline{\rho}^{+} \underline{\rho}^{-}}{\underline{\rho}^{-} \mathrm{th}^{+}+\underline{\rho}^{+} \mathrm{th}^{-}} \frac{\left(\llbracket \mathbf{c}^{ \pm} \rrbracket \cdot D\right)^{2}}{|D|} .
$$

\subsection{Kelvin-Helmholtz instabilities for the linearized two-fluids equations}

A brief look at (22) shows that the Fourier modes $(\mathscr{F} \dot{\zeta}(\mathbf{k}), \mathscr{F} \dot{\psi}(\mathbf{k}))$ are stable (i.e. they are not exponentially ${ }^{2}$ amplified) if and only if

$$
g^{\prime}-e(\mathbf{k})+\frac{\sigma}{\rho^{+}+\rho^{-}}|\mathbf{k}|^{2}>0
$$

From the explicit expression of $e(\mathbf{k})$ stemming from (23), the most unstable direction corresponds to $\mathbf{k}$ parallel to $\llbracket c^{ \pm} \rrbracket$, and we therefore restrict our stability analysis to wave numbers $\mathbf{k}$ oriented along this most unstable direction. Without loss of generality, we can assume that

$$
\llbracket \mathbf{c}^{ \pm} \rrbracket=\llbracket c^{ \pm} \rrbracket \mathbf{e}_{x}, \quad\left(\llbracket c^{ \pm} \rrbracket=\left|\llbracket \mathbf{c}^{ \pm} \rrbracket\right|\right)
$$

and we consider therefore the stability of the Fourier modes $(\mathscr{F} \dot{\zeta}(\mathbf{k}), \mathscr{F} \dot{\psi}(\mathbf{k}))$ corresponding to $\mathbf{k}=k \mathbf{e}_{x}$, with $k=|\mathbf{k}|$.

From (23), we deduce easily that for all $\mathbf{k}=k \mathbf{e}_{x}$,

\footnotetext{
${ }^{2}$ The endpoint case $g^{\prime}-e(\mathbf{k})=0$ actually corresponds to a linear amplification.
} 


$$
g^{\prime}-e(\mathbf{k})+\frac{\sigma}{\rho^{+}+\rho^{-}}|\mathbf{k}|^{2}>0 \Longleftrightarrow \llbracket c^{ \pm} \rrbracket^{2}<\Omega_{K H} \alpha_{\sigma}(k),
$$

where we used the notations

$$
\begin{aligned}
\Omega_{K H} & =\frac{g^{\prime} H_{0}}{\underline{\rho}^{+}} \quad \underline{\rho}^{-} \quad\left(\text { and } H_{0}=\underline{\rho}^{-} H^{+}+\underline{\rho}^{+} H^{-}\right), \\
\alpha_{\sigma}(k) & =\left(1+\frac{\sigma}{g^{\prime}\left(\rho^{+}+\rho^{-}\right)} k^{2}\right) \alpha(k), \\
\alpha(k) & =\underline{\rho}^{-} \frac{\mathrm{th}^{+}}{H_{0} k}+\underline{\rho}^{+} \frac{\mathrm{th}^{-}}{H_{0} k} .
\end{aligned}
$$
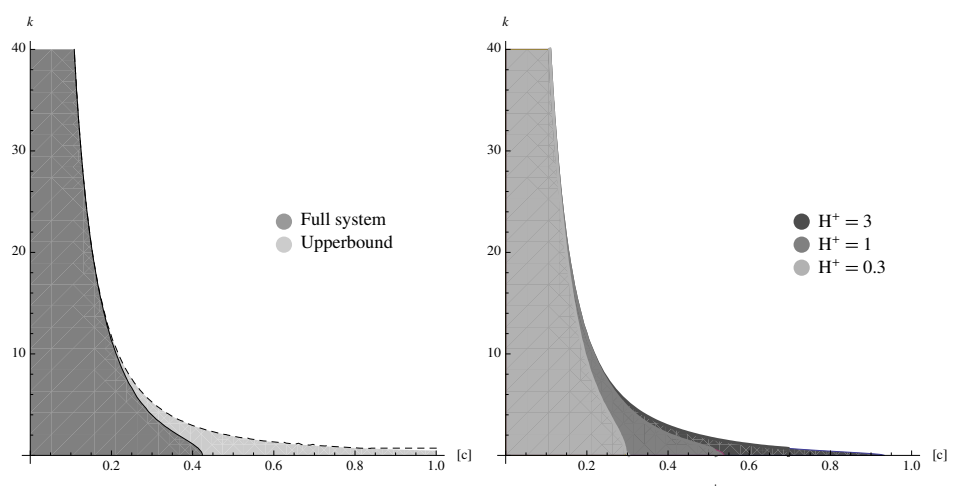

(a) An upper bound for the stable area

(b) Influence of $\mathrm{H}^{+}$on the stable area.

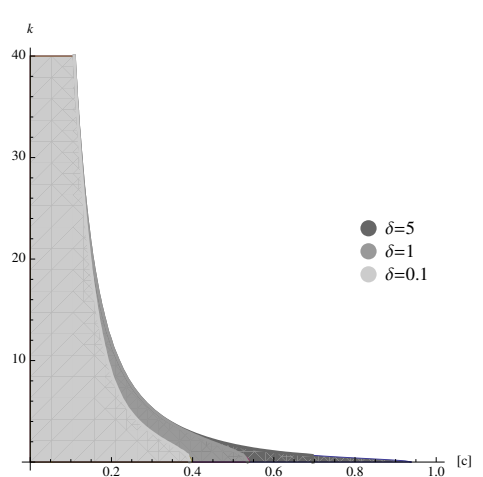

(c) Influence of the depth ratio $\delta$.

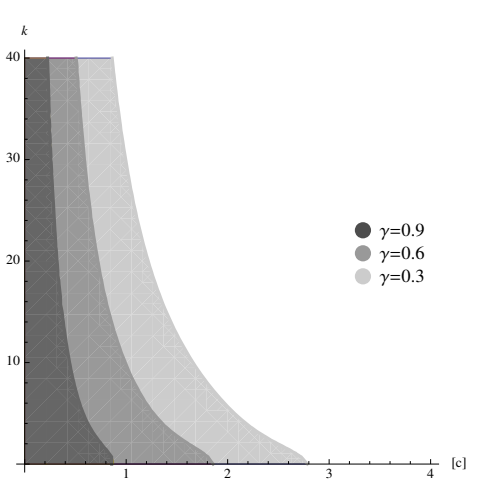

(d) Influence of the density ratio $\gamma$.

Fig. 3 Surface tension is neglected $(\sigma=0)$. Unless otherwise specified, the parameters are $\delta=$ $0.15 / 0.62, H^{+}=0.62, \gamma=0.494 / 0.506$ and $g=9.81$. We write $\llbracket c^{ \pm} \rrbracket$ simply as $[c]$ in all the figures through this paper. 
Since $\alpha(\cdot)$ is a decreasing function on $\mathbb{R}^{+}$with maximal value 1 at the origin, the modes with low wavenumber are stable if and only if

$$
\text { (Stab 1) } \quad \llbracket c^{ \pm} \rrbracket^{2}<\Omega_{K H} ;
$$

if it is satisfied, there is a unique $k_{K H} \in \mathbb{R}_{*}^{+} \cup\{+\infty\}$ for each $\llbracket c^{ \pm} \rrbracket$, such that all the modes corresponding to $0 \leq k<k_{K H}$ are stable,

$$
\text { (Critical wavenumber) } \quad k_{K H}=\min \alpha_{\sigma}^{-1}\left(\frac{\llbracket c^{ \pm} \rrbracket^{2}}{\Omega_{K H}}\right) ;
$$

the quantity $k_{K H}$ is therefore the critical wavenumber above which Kelvin-Helmholtz instabilities appear for the perturbation of the constant shear flow. Since one obviously has

$$
\alpha(k) \leq \alpha_{\infty}(k):=\frac{1}{k H_{0}},
$$

one obtains the following upper bound for $k_{K H}$ (see Figure3(a)),

$$
k_{K H} \leq k_{K H}^{\infty}:=\min \alpha_{\sigma, \infty}^{-1}\left(\frac{\llbracket c^{ \pm} \rrbracket^{2}}{\Omega_{K H}}\right)
$$

where $\alpha_{\sigma, \infty}(k)=\left(1+\frac{\sigma}{g^{\prime}\left(\rho^{+}+\rho^{-}\right)} k^{2}\right) \alpha_{\infty}(k)$. Direct computations yield

$$
\begin{array}{r}
k_{K H}^{\infty}=\frac{\llbracket c^{ \pm} \rrbracket^{2}}{\Omega_{K H}} \frac{\text { Bo }}{2 H_{0}}\left(1-\sqrt{1-4 \frac{\Omega_{K H}^{2}}{\mathrm{Bo} \llbracket c^{ \pm} \rrbracket^{4}}}\right) \quad \text { (with surface tension) }, \\
k_{K H}^{\infty}=\frac{\Omega_{K H}}{\llbracket c^{ \pm} \rrbracket^{2}} \frac{1}{H_{0}} \quad \text { (without surface tension) } .
\end{array}
$$

Note that this upper bound coincides with the critical wavenumber $k_{K H}$ when both fluid layers are of infinite depth $\left(H^{+}=H^{-}=\infty\right)$.

It is straightforward to analyze the influence on the stable area (i.e. the set all the couples $\left(\llbracket c^{ \pm} \rrbracket, k\right)$ such that (24) is satisfied) of the depth $H^{+}$, the depth ratio $\delta=H^{-} / H^{+}$and the density ratio $\gamma=\rho^{-} / \underline{\rho}^{+}$. One can see from (24) that, when the surface tension is neglected $(\sigma=0)$, the interface problem is more stable when $H^{+}$increases, $\delta$ increases, or $\gamma$ decreases (see Figures 3(b), 3(c), 3(d) in the case without surface tension).

Remark 3. We chose to present the linear stability criteria derived in this paper in terms of $\llbracket c^{ \pm} \rrbracket$ to make the comparison with the nonlinear criterion (1) more transparent. Another possibility, often used in the literature, would have been to use instead the (dimensionless) Froude number

$$
\mathrm{Fr}=\frac{\llbracket c^{ \pm} \rrbracket}{\sqrt{g^{\prime} H_{0}}}
$$

Using this notation, the criterion (Stab 1) derived above can be restated as 


$$
\operatorname{Fr}<\left(\underline{\rho}^{+} \underline{\rho}^{-}\right)^{-1 / 2}
$$

the right-hand-side depends only on the density ratio of the two layers, and is always greater than 2. This indicates that this stability criterion is quite reasonable, even when the density ratio goes to 1 , even though $g^{\prime}$ goes to zero. This approach is consequently used in $\S 6.2$ to study the rigid limit limit in the case of two layers with a free surface.

In absence of surface tension $(\sigma=0), \alpha_{\sigma}(\cdot)=\alpha(\cdot)$ is a decreasing function and goes to zero at infinity. One then easily deduces that in presence of a nonzero shear $\llbracket \mathbf{c}^{ \pm} \rrbracket$ there are always unstable modes. In presence of surface tension, the situation is different. If (Stab 1) is satisfied, then there the modes with low wave numbers are stable; since $\alpha_{\sigma}(\cdot)$ grow to infinity at infinity, one directly infers from (24) that modes with high wavenumbers are stable. It is then possible to have stability of all Fourier modes if the shear does not exceed a critical value $\llbracket c^{ \pm} \rrbracket=\sqrt{\Omega^{c r}}$; more precisely, all modes are stable if and only if

(Stab 2)

$$
\llbracket c^{ \pm} \rrbracket^{2} \leq \Omega^{c r}:=\Omega_{K H} \min _{\mathbb{R}^{+}} \alpha_{\sigma}
$$

(note that this criterion can be understood as the linear approximation of (1)). When $\sigma=0$, one gets $\Omega^{c r}=0<\Omega_{K H}$; in presence of surface tension, $\Omega^{c r} \leq \Omega_{K H}$ and equality holds if the capillary effects are strong enough (see Figure 4 ), in which case the existence of some stable modes implies that all modes are stable.

There does not seem to be any simple explicit formula for the critical value $\Omega^{c r}$. However, it is possible to give upper and lower bounds for this quantity. Note that these bounds are very precise in most situations (for the experiment studied in $\S 4.3$ below for instance, there is an error of only $3 \%$ between $\Omega_{-}^{c r}$ and $\Omega_{+}^{c r}$ ). We also point

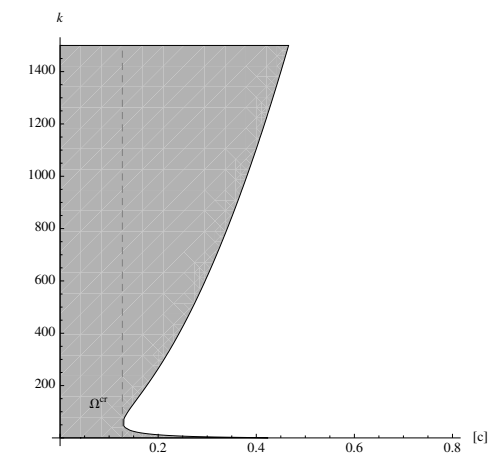

(a) $\Omega^{c r}<\Omega_{K H}$ (here, $H^{+}=0.62$ ).

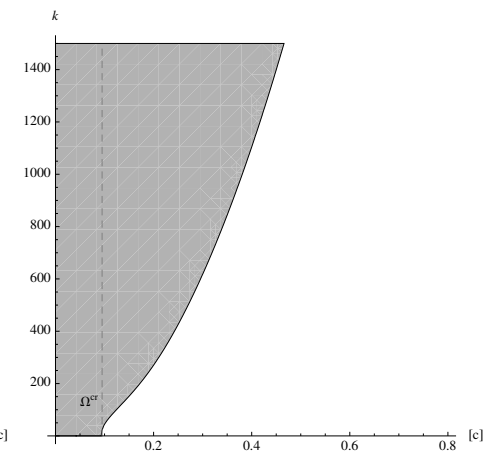

(b) $\Omega^{c r}=\Omega_{K H}$ (here, $\left.H^{+}=0.03\right)$.

Fig. 4 Stable area with surface tension. Parameters are $\delta=0.15 / 0.62, \gamma=0.494 / 0.506, \rho^{+}+$ $\rho^{-}=2021, \sigma=0.073$ and $g=9.81$. 
out that the upper bound given in the proposition coincides with Kelvin's criterion derived in the setting $H^{+}=H^{-}=\infty$ [23].

Proposition 1. One has

$$
\Omega_{-}^{c r}<\Omega^{c r}<\Omega_{+}^{c r}
$$

with

$$
\Omega_{-}^{c r}=\Omega_{K H} \alpha\left(\frac{1}{2 H_{0}} \sqrt{\mathrm{Bo}}\right), \quad \Omega_{+}^{c r}=\Omega_{K H} \frac{2}{\sqrt{\mathrm{Bo}}},
$$

where $\alpha(\cdot)$ is as in (27) and the Bond number Bo as defined in (5).

Remark 4. Note that the approximation $\Omega^{c r} \sim \Omega_{+}^{c r}$ is in perfect agreement with the nonlinear criterion (1) derived in [32], namely,

$$
-\llbracket \partial_{z} P_{\text {linterf }}^{ \pm} \rrbracket>\frac{1}{4} \frac{\left(\rho^{+} \rho^{-}\right)^{2}}{\sigma\left(\rho^{+}+\rho^{-}\right)^{2}} \mathfrak{c}(\zeta)\left|\llbracket \mathbf{U}_{\text {linterf }}^{ \pm} \rrbracket\right|_{\infty}^{4} .
$$

Indeed, replacing $\mathbf{U}_{\text {linterf }}^{ \pm}$by $c^{ \pm}$in the above formula, and replacing the pressure by its hydrostatic approximation $\partial_{z} P^{ \pm}=-\rho^{ \pm} g$, this nonlinear criterion yields

$$
\llbracket c^{ \pm} \rrbracket^{2}<\mathfrak{c}(\zeta)^{-1 / 2} \Omega_{+}^{c r}
$$

Sticking to the linear theory as we do in this article allows us to carry the computations further and to get an explicit expression for the constant $\mathfrak{c}(\zeta)$ (or more precisely of its equivalent for the linear theory); we get in particular from the above proposition some upper and lower bounds for $\mathfrak{c}(\zeta$ ) (for the experiment considered in $\S 4.3$, one deduces that $\mathfrak{c}(\zeta) \sim 1)$.

Proof. Let us first prove a lower bound $\alpha_{\sigma}^{-}$to $\alpha_{\sigma}$. Let $k_{0}>0$. One has obviously $\alpha_{\sigma}(k) \geq \alpha(k)$ for all $k \geq 0$ and, since $\alpha(\cdot)$ is a decreasing function, we get

$$
\forall 0 \leq k \leq k_{0}, \quad \alpha(k) \geq \alpha\left(k_{0}\right)
$$

For $k \geq k_{0}$, let us remark first that

$$
g^{\prime}+\frac{\sigma}{\rho^{+}+\rho^{-}} k^{2} \geq \beta k
$$

if $\beta>0$ is small enough. The largest possible value for $\beta$ is $\beta=2 \frac{\sqrt{g^{\prime} \sigma}}{\sqrt{\rho^{+}+\rho^{-}}}$, so that

$$
\alpha_{\sigma}(k)=\left(1+\frac{\sigma}{g^{\prime}\left(\rho^{+}+\rho^{-}\right)} k^{2}\right) \alpha(k) \geq 2 \frac{\sqrt{\sigma}}{\sqrt{g^{\prime}\left(\rho^{+}+\rho^{-}\right)}} k \alpha(k)
$$

Since the function $k \mapsto k \alpha(k)$ is increasing on $\mathbb{R}^{+}$, we get that

$$
\forall k \geq k_{0}, \quad \alpha_{\sigma}(k) \geq 2 \frac{\sqrt{\sigma}}{\sqrt{g^{\prime}\left(\rho^{+}+\rho^{-}\right)}} k_{0} \alpha\left(k_{0}\right) .
$$


It follows from the above that

$$
\forall k \geq 0, \quad \alpha_{\sigma}(k) \geq \min \left\{\alpha\left(k_{0}\right), 2 \frac{\sqrt{\sigma}}{\sqrt{g^{\prime}\left(\rho^{+}+\rho^{-}\right)}} k_{0} \alpha\left(k_{0}\right)\right\} .
$$

We now choose $k_{0}$ such that the two quantities in the right-hand-side are equal, namely,

$$
k_{0}=\frac{1}{2} \frac{\sqrt{g^{\prime}\left(\rho^{+}+\rho^{-}\right)}}{\sqrt{\sigma}}
$$

to get

$$
\forall k \geq 0, \quad \alpha_{\sigma}(k) \geq \alpha_{\sigma}^{-}, \quad \text { with } \quad \alpha_{\sigma}^{-}=\alpha\left(\frac{1}{2} \frac{\sqrt{g^{\prime}\left(\rho^{+}+\rho^{-}\right)}}{\sqrt{\sigma}}\right)
$$

We now turn to derive an upper bound $\alpha_{\sigma}^{+}$for the minimum of $\alpha_{\sigma}$. It is obtained simply by observing that $\alpha(k) \leq\left(k H_{0}\right)^{-1}$. This yields

$$
\alpha_{\sigma}(k) \leq \frac{1}{k H_{0}}\left(1+\frac{\sigma}{g^{\prime}\left(\rho^{+}+\rho^{-}\right)} k^{2}\right)
$$

and an upper bound for the minimum of $\alpha_{\sigma}$ is therefore given by the minimum of the right-hand-side

$$
\forall k \geq 0, \quad \alpha_{\sigma}(k) \leq \alpha_{\sigma}^{+}, \quad \text { with } \quad \alpha_{\sigma}^{+}=\frac{2}{H_{0}} \frac{\sqrt{\sigma}}{\sqrt{g^{\prime}\left(\rho^{+}+\rho^{-}\right)}} .
$$

The expressions for $\Omega_{ \pm}^{c r}$ follow easily from $\alpha_{\sigma}^{ \pm}$.

\section{Shallow water approximations in the rigid lid case}

The goal of this section is to investigate whether shallow water models are able to give a good account of the formation of Kelvin-Helmholtz instabilities for twofluids interfaces, in the rigid lid case. We investigate several shallow water models (some of them new) and study the same stability issues as in $\S 3$ for the full Euler equations. This allows us to give some insight on the ability of these asymptotic models to describe the Kelvin-Helmholtz instabilities correctly.

Notation: When the fluid layers are both of finite depth, the first equation in (11) can equivalently be written under the form (conservation of mass)

$$
\partial_{t} \zeta \pm \nabla \cdot\left(H^{ \pm}(\zeta) \bar{V}^{ \pm}\right)=0, \quad \text { with } \quad H^{ \pm}(\zeta)=H^{ \pm} \pm \zeta
$$

and where $\bar{V}^{ \pm}$is the vertically averaged horizontal velocity, 


$$
\bar{V}^{ \pm}(t, X)= \pm \frac{1}{H^{ \pm}(\zeta)} \int_{\mp H^{ \pm}}^{\zeta} V^{ \pm}(t, X, z) d z
$$

Shallow water approximations consist in writing the second equation of (11) as an approximate evolution equation on $\bar{V}^{ \pm}$. In order to do so, $U_{\|}^{ \pm}$is related to $\bar{V}^{ \pm}$through an asymptotic expansion in terms of the shallowness parameter $\mu^{ \pm}=\left(H^{ \pm}\right)^{2} / L^{2}$, where $L$ is the typical horizontal length of the interfacial waves (see for instance [8] for a systematic approach). With a first order expansion, one obtains the Shallow water/Shallow water system (SW/SW) described in $\S 4.1$, while a second order expansion gives the Green-Naghdi/Green-Naghdi system (GN/GN) in $\S 4.2$. We also consider in $\S 4.2 .4$ and $\S 4.2 .5$ two classes of "regularized" models. We show in particular that these news models allow one to reproduce the same values for the critical shear as for the full Euler equations (which is not the case with the standard GN/GN model, even in presence of surface tension). Finally, we apply in $\S 4.3$ some of our results to experiments reported in [29].

\subsection{First order approximation}

We consider here the first order approximation under the Shallow Water/Shallow Water regime. We derive the so called Shallow Water/Shallow Water system first, and then the system is linearized near a constant shear flow. The stability criterion is obtained as in the full system case.

\subsubsection{The Shallow Water/Shallow Water equations}

Up to terms that are $O(\mu)$ times smaller, one can write

$$
U_{\|}^{ \pm} \sim \bar{V}^{ \pm} \quad \text { and } \quad \underline{w}^{ \pm} \sim 0
$$

(see for instance $[41,14,15,34,8]$ ), and the second equation of (11) can be written at leading order under the form

$$
\partial_{t} \bar{V}^{ \pm}+g \nabla \zeta+\frac{1}{2} \nabla\left|\bar{V}^{ \pm}\right|^{2}=-\frac{1}{\rho^{ \pm}} \nabla \underline{P}^{ \pm} .
$$

The first order shallow water approximation of (11) is then given by

$$
\left\{\begin{array}{l}
\partial_{t} \zeta \pm \nabla \cdot\left(H^{ \pm}(\zeta) \bar{V}^{ \pm}\right)=0, \\
\partial_{t} \bar{V}^{ \pm}+g \nabla \zeta+\frac{1}{2} \nabla\left|\bar{V}^{ \pm}\right|^{2}=-\frac{1}{\rho^{ \pm}} \nabla \underline{P}^{ \pm}
\end{array}\right.
$$

with

$$
H^{ \pm}(\zeta)=H^{ \pm} \pm \zeta
$$


Remark 5. As in Remark 1 for the full equations, one can derive a reduced formulation of (11) in terms of $\zeta$ and $\bar{V}=\underline{\rho}^{+} \bar{V}^{+}-\underline{\rho}^{-} \bar{V}^{-}$. In dimension $d=1$ (writing $\bar{v}=\bar{V})$ this system is

$$
\left\{\begin{array}{l}
\partial_{t} \zeta+\partial_{x}[H(\zeta) \bar{v}]=0 \\
\partial_{t} \bar{v}+g^{\prime} \partial_{x} \zeta+\frac{1}{2} \partial_{x}\left[H^{\prime}(\zeta) \bar{v}^{2}\right]=-\frac{\sigma}{\rho^{+}+\rho^{-}} \kappa(\zeta)
\end{array}\right.
$$

with

$$
H(\zeta)=\frac{H^{-}(\zeta) H^{+}(\zeta)}{\underline{\rho}^{+} H^{-}(\zeta)+\underline{\rho}^{-} H^{+}(\zeta)}
$$

in dimension $d=2$, one can also obtain a reduced formulation, but it is more complicated and involves non local operators [8,30]. Note also that this model can be simplified under the so called Boussinesq approximation when the two densities are very close [9].

\subsubsection{Linearization around a constant shear $\left(\mathrm{c}^{+}, \mathrm{c}^{-}\right)$}

We consider here the linear equations governing small perturbations of the constant horizontal shear $\zeta=0, V^{ \pm}=\mathbf{c}^{ \pm}$(and therefore $\bar{V}^{ \pm}=\mathbf{c}^{ \pm}$).

Linearizing (29) around $\zeta=0, V^{ \pm}=\mathbf{c}^{ \pm}$, we find the following equations for the perturbations $\left(\dot{\zeta}, \dot{V}^{ \pm}\right)$,

$$
\left\{\begin{array}{l}
\partial_{t} \dot{\zeta}+\mathbf{c}^{ \pm} \cdot \nabla \dot{\zeta} \pm H^{ \pm} \nabla \cdot \dot{V}^{ \pm}=0 \\
\partial_{t} \dot{V}^{ \pm}+g \nabla \dot{\zeta}+\mathbf{c}^{ \pm} \cdot \nabla \dot{V}^{ \pm}=-\frac{1}{\rho^{ \pm}} \nabla \underline{P}^{ \pm}
\end{array}\right.
$$

note that since the flow is assumed to be potential at leading order in order to derive the nonlinear shallow water equations (30), we restrict to perturbations $\dot{V}^{ \pm}$such that $\nabla^{\perp} \cdot \dot{V}^{ \pm}=0$.

In the same spirit as in Remark 1, we can derive a reduced formulation for these equations; denoting $\dot{V}=\underline{\rho}^{+} \dot{V}^{+}-\underline{\rho}^{-} \dot{V}^{-}$, we first get

$$
\left\{\begin{array}{l}
\partial_{t} \dot{\zeta}+\mathbf{c}^{ \pm} \cdot \nabla \dot{\zeta} \pm H^{ \pm} \nabla \cdot \dot{V}^{ \pm}=0, \\
\partial_{t} \dot{V}+\left\langle\mathbf{c}^{ \pm}\right\rangle \cdot \nabla \dot{V}+g^{\prime} \nabla \dot{\zeta}+\llbracket \mathbf{c}^{ \pm} \rrbracket \cdot \nabla\left\langle\underline{\rho}^{ \pm} \dot{V}^{ \pm}\right\rangle=\frac{\sigma}{\rho^{+}+\rho^{-}} \Delta \nabla \dot{\zeta}
\end{array}\right.
$$

in order to put this system in a closed system of equations on $(\dot{\zeta}, \dot{V})$, we need the following lemma.

Lemma 2. One has, with $H_{0}=\underline{\rho}^{+} H^{-}+\underline{\rho}^{-} H^{+}$,

$$
\dot{V}^{+}=\frac{H^{-}}{H_{0}} \dot{V}-\frac{\rho^{-}}{H_{0}} \Pi\left(\llbracket \mathbf{c}^{ \pm} \rrbracket \dot{\zeta}\right), \quad \dot{V}^{-}=-\frac{H^{+}}{H_{0}} \dot{V}-\frac{\rho^{+}}{H_{0}} \Pi\left(\llbracket \mathbf{c}^{ \pm} \rrbracket \dot{\zeta}\right)
$$


where $\Pi=\frac{\nabla \nabla^{T}}{\Delta}$ is the projector onto gradient vector fields.

Proof. From the first equation of (31), we get

$$
H^{+} \nabla \cdot \dot{V}^{+}+H^{-} \nabla \cdot \dot{V}^{-}=-\llbracket \mathbf{c} \rrbracket \cdot \nabla \dot{\zeta}
$$

recalling that the perturbations $\dot{V}^{ \pm}$are such that $\nabla^{\perp} \cdot \dot{V}^{ \pm}=0$, we get after integrating in space that

$$
H^{+} \dot{V}^{+}+H^{-} \dot{V}^{-}=-\Pi(\llbracket \mathbf{c} \rrbracket \dot{\zeta})
$$

Together with the definition of $\dot{V}$, namely,

$$
\underline{\rho}^{+} \dot{V}^{+}-\underline{\rho}^{-} \dot{V}^{-}=\dot{V}
$$

this yields a system on $\dot{V}^{ \pm}$whose solution is provided by the formulas stated in the lemma.

Using the lemma, we can rewrite (31) under the form as a system of $(\dot{\zeta}, \dot{V})$

$$
\left\{\begin{array}{l}
\partial_{t} \dot{\zeta}+\left(\mathbf{c}^{+}-\underline{\rho^{-}} \frac{H^{+}}{H_{0}} \llbracket \mathbf{c}^{ \pm} \rrbracket\right) \cdot \nabla \dot{\zeta}+\frac{H^{+} H^{-}}{H_{0}} \nabla \cdot \dot{v}=0 \\
\partial_{t} \dot{V}+\left(\mathbf{c}^{+}-\underline{\rho}^{-} \frac{H^{+}}{H_{0}} \llbracket \mathbf{c}^{ \pm} \rrbracket\right) \cdot \nabla \dot{V} \\
\quad+\left(g^{\prime}-\frac{\underline{\rho}^{+} \underline{\rho}^{-}}{H_{0}} \frac{\left(\llbracket \mathbf{c}^{ \pm} \rrbracket \cdot D\right)^{2}}{D^{2}}-\frac{\sigma}{\rho^{+}+\rho^{-}} \Delta\right) \nabla \dot{\zeta}=0 .
\end{array}\right.
$$

\subsubsection{The SW/SW model and the Kelvin-Helmholtz instabilities}

In $\S 3.2$ we derived a stability condition (Stab 1) ensuring the existence of stable modes with low wave number, derived the expression of the critical wave number at the boundary of the stability area, and derived a stronger stability condition (Stab 2) ensuring the stability of all modes. We derive here similar conditions for the SW/SW model, which allows us to conclude that this model underestimates the Kelvin-Helmholtz instabilities.

A brief look at (32) shows that the Fourier modes $(\mathscr{F} \dot{\zeta}(\mathbf{k}), \mathscr{F} \dot{\psi}(\mathbf{k}))$ are stable if and only if

$$
g^{\prime}-\frac{\underline{\rho}^{+}}{\underline{\rho}^{-}} \frac{\left(\llbracket \mathbf{c}^{ \pm} \rrbracket \cdot \mathbf{k}\right)^{2}}{|\mathbf{k}|^{2}}+\frac{\sigma}{\rho^{+}+\rho^{-}}|\mathbf{k}|^{2}>0 .
$$

As for the full equations in $\S 3.2$, the most unstable modes are those for which $\mathbf{k}$ is parallel to the shear, and we again focus on these most unstable modes. As in $\S 3.2$, we write $\llbracket \mathbf{c}^{ \pm} \rrbracket=\llbracket c^{ \pm} \rrbracket \mathbf{e}_{x}$ and $\mathbf{k}=k \mathbf{e}_{x}$, with $\llbracket c^{ \pm} \rrbracket=\left|\llbracket \mathbf{c}^{ \pm} \rrbracket\right|$ and $k=|\mathbf{k}|$. We readily get that a Fourier mode corresponding to the wavenumber $\mathbf{k}$ is stable for (32) if and only if 


$$
g^{\prime}\left(1-\frac{\llbracket c^{ \pm} \rrbracket^{2}}{\Omega_{K H}}\right)+\frac{\sigma}{\rho^{+}+\rho^{-}} k^{2}>0 \Longleftrightarrow \llbracket c^{ \pm} \rrbracket^{2}<\Omega_{K H}\left(1+\frac{\sigma}{g^{\prime}\left(\rho^{+}+\rho^{-}\right)} k^{2}\right) .
$$

It follows easily that the modes with low wavenumber are stable if and only if

$$
(\operatorname{Stab} 1)_{S W} \quad \llbracket c^{ \pm} \rrbracket^{2}<\Omega_{K H},
$$

which is exactly the same as the condition (Stab 1) derived for the full equations in $\S 3.2$. If it is satisfied, then all the modes are stable, so that in the SW/SW model, we have for each $\llbracket c^{ \pm} \rrbracket$

$$
(\text { Critical wavenumber })_{S W} \quad k_{S W}=+\infty,
$$

and the critical shear $\sqrt{\Omega_{S W}^{c r}}$ below which all modes are stable coincides with $\sqrt{\Omega_{K H}}$ : all modes are stable if and only if

$$
(\operatorname{Stab} 2)_{S W} \quad \llbracket c^{ \pm} \rrbracket^{2} \leq \Omega_{S W}^{c r} \quad \text { with } \quad \Omega_{S W}^{c r}=\Omega_{K H} .
$$

This condition differs in general from (Stab 2) derived in $\S 3.2$ for the full equation since we always have $\Omega^{c r} \leq \Omega_{K H}$ and, in general, $\Omega^{c r}<\Omega_{K H}$ (this is the case for small capillary effects, see Figure 4). Therefore, the SW/SW model underestimates Kelvin-Helmholtz instabilities.

Remark 6. The full, nonlinear, analysis can be carried out for the SW/SW equations. This has been done in [30] and improved in [10]; in particular, the SW/SW equations (29) (or equivalently, (30) when $d=1$ ) are locally well-posed provided that the initial condition $\left(\zeta^{0}, \bar{V}^{ \pm, 0}\right)$ satisfies

$$
\llbracket \bar{V}^{ \pm, 0} \rrbracket^{2}<\Omega_{K H}\left(\zeta^{0}\right) \quad \text { with } \quad \Omega_{K H}\left(\zeta^{0}\right)=\frac{g^{\prime}\left(\underline{\rho}^{-} H^{-}\left(\zeta^{0}\right)+\underline{\rho}^{+} H^{+}\left(\zeta^{0}\right)\right)}{\underline{\rho}^{+} \underline{\rho}^{-}},
$$

and $H^{ \pm}\left(\zeta^{0}\right)=H^{ \pm} \pm \zeta^{0}$. The stability condition (Stab 1) $)_{S W}$ is therefore the condition one naturally expects from the condition above when linearizing around the constant shear $\left(\zeta=0, \bar{V}^{ \pm}=\mathbf{c}^{ \pm}\right)$.

\subsection{Second order approximation}

This section is devoted to the second order approximation under the SW/SW regime, which is the so called Green-Naghdi/Green-Naghdi (or GN/GN) system. We linearize the system again near a constant shear flow. A different stability criterion is then obtained based on the linearized system. Besides, we also derive and discuss two "regularized" GN/GN models in order to get a better approximation on the critical shear and therefore a better description of the formation of Kelvin-Helmholtz instabilities. 


\subsubsection{The Green-Naghdi/Green-Naghdi equations}

We proceed as in $\S 4.1$, but go one step further in the asymptotic expression of $\dot{V}^{ \pm}$ in terms of $\bar{V}^{ \pm}$, namely

$$
U_{\|}^{ \pm} \sim\left(1+\mathscr{T}^{ \pm}\right) \bar{V}^{ \pm} \quad \text { and } \quad \underline{w}^{ \pm} \sim \mp H^{ \pm}(\zeta) \partial_{x} \bar{V}^{ \pm}
$$

where

$$
\mathscr{T}^{ \pm} \bullet=-\frac{1}{3 H^{ \pm}(\zeta)} \nabla\left(H^{ \pm}(\zeta)^{3} \nabla \cdot \bullet\right) \quad \text { with } \quad H^{ \pm}(\zeta)=H^{ \pm} \pm \zeta
$$

(we refer to $[15,34]$ for the derivation of this asymptotic expansion).

Putting the first equation of (11) under the equivalent form (28), and plugging the above approximations for $U_{\|}^{ \pm}$and $\underline{w}^{ \pm}$into the second equation of (11), we obtain the second order shallow water approximation $[36,15,18]$ from which many other asymptotic models can be derived [27],

$$
\left\{\begin{array}{l}
\partial_{t} \zeta \pm \nabla \cdot\left(H^{ \pm}(\zeta) \bar{V}^{ \pm}\right)=0 \\
\left(1+\mathscr{T}^{ \pm}\right) \partial_{t} \bar{V}^{ \pm}+g \nabla \zeta+\frac{1}{2} \nabla\left|\bar{V}^{ \pm}\right|^{2}+Q^{ \pm}=-\frac{1}{\rho^{ \pm}} \nabla \underline{P}^{ \pm}
\end{array}\right.
$$

with $\llbracket \underline{P}^{ \pm} \rrbracket=\sigma \kappa(\zeta)$ and

$$
Q^{ \pm}\left(\bar{V}^{ \pm}\right)=-\frac{1}{3 H^{ \pm}(\zeta)} \nabla\left(H^{ \pm}(\zeta)^{3}\left(\bar{V}^{ \pm} \cdot \nabla\left(\nabla \cdot \bar{V}^{ \pm}\right)-\left|\nabla \cdot \bar{V}^{ \pm}\right|^{2}\right)\right) .
$$

In the one fluid case $\rho^{-}=0$, these equations coincide with the Green-Naghdi (or Serre) equations; adopting the terminology of [8], we therefore refer to these equations as the Green-Naghdi/Green-Naghdi (or GN/GN) equations. As shown in [15] these equations have a (non-canonical) Hamiltonian structure.

\subsubsection{Linearization around a constant shear $\left(c^{+}, c^{-}\right)$}

Though it is possible to work with the two dimensional case $d=2$ as in $\S 4.1$, we focus our interest here on the one dimensional case $d=1$ for the sake of clarity. We therefore denote by $v$ the horizontal velocity instead of $V$ (and similarly, we write $\bar{v}$, etc). Linearizing (35) around $\zeta=0, \bar{v}^{ \pm}=c^{ \pm}$, we find the following equations,

$$
\left\{\begin{array}{l}
\partial_{t} \dot{\zeta}+c^{ \pm} \partial_{x} \dot{\zeta} \pm H^{ \pm} \partial_{x} \dot{v}^{ \pm}=0 \\
\left(1-\frac{1}{3}\left(H^{ \pm}\right)^{2} \partial_{x}^{2}\right)\left(\partial_{t} \dot{v}^{ \pm}+c^{ \pm} \partial_{x} \dot{v}^{ \pm}\right)+g \partial_{x} \dot{\zeta}=-\frac{1}{\rho^{ \pm}} \partial_{x} \underline{P}^{ \pm} .
\end{array}\right.
$$

Using the formulas of Lemma 2, we can rewrite the second equation under the form 


$$
\left\{\begin{array}{l}
\partial_{t} \dot{\zeta}+\left(c^{+}-\underline{\rho^{-}} \frac{H^{+}}{H_{0}} \llbracket c^{ \pm} \rrbracket\right) \partial_{x} \dot{\zeta}+\frac{H^{+} H^{-}}{H_{0}} \partial_{x} \dot{v}=0, \\
\left(1-\frac{1}{3}\left(H^{ \pm}\right)^{2} \partial_{x}^{2}\right)\left(\left(\partial_{t}+c^{ \pm} \partial_{x}\right)\left( \pm \frac{H^{\mp}}{H_{0}} \dot{v}-\frac{\underline{\rho}^{\mp}}{H_{0}} \llbracket c^{ \pm} \rrbracket \dot{\zeta}\right)\right)+g \partial_{x} \dot{\zeta}=-\frac{1}{\rho^{ \pm}} \partial_{x} \underline{P}^{ \pm}
\end{array}\right.
$$

Eliminating the pressure from these equations, we get after some computations,

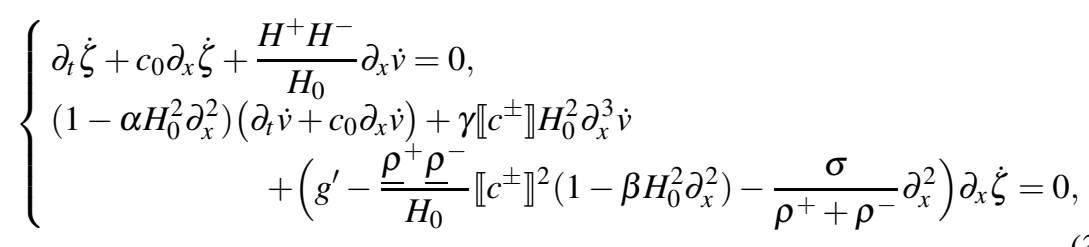

with

$$
\begin{gathered}
c_{0}=\left(c^{+}-\underline{\rho^{-}} \frac{H^{+}}{H_{0}} \llbracket c^{ \pm} \rrbracket\right), \quad \alpha=\frac{1}{3}\left(\underline{\rho}^{+} H^{+}+\underline{\rho}^{-} H^{-}\right) \frac{H^{+} H^{-}}{H_{0}^{3}} \\
\beta=\frac{\underline{\rho}^{-}\left(H^{+}\right)^{3}+\underline{\rho}^{+}\left(H^{-}\right)^{3}}{3 H_{0}^{3}}, \quad \gamma=-\frac{2}{3} \frac{\underline{\rho}^{+} \underline{\rho}^{-}}{H_{0}}\left(\left(H^{+}\right)^{2}-\left(H^{-}\right)^{2}\right) \frac{H^{+} H^{-}}{H_{0}^{3}} .
\end{gathered}
$$

\subsubsection{The GN/GN model and the Kelvin-Helmholtz instabilities}

As in $\S 4.1 .3$ with the SW/SW model, we want to study the linear stability of (36) and compare it with the behavior of the full equations investigated in $\S 3.2$.

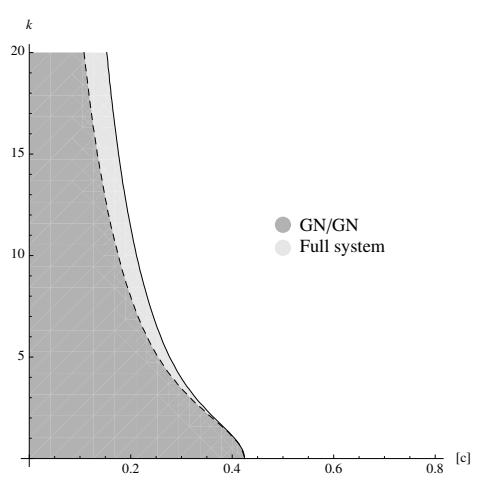

(a) Without surface tension.

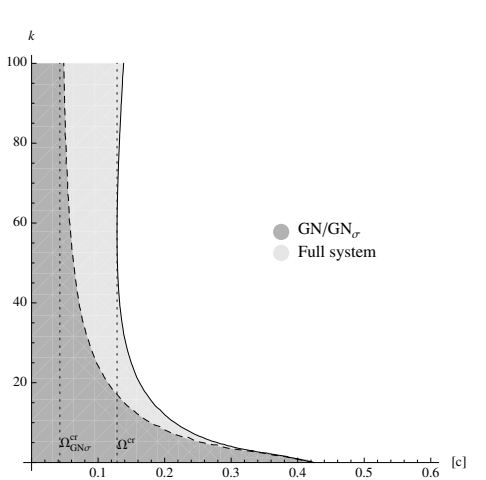

(b) With surface tension.

Fig. 5 Stables areas for the full system (Euler) and the GN/GN model without or with surface tension $(\sigma=0.073)$. Parameters $\rho^{+}+\rho^{-}=2021, \gamma=0.494 / 0.506, H^{+}=0.62, \delta=0.15 / 0.62$ and $g=9.81$. 
With a simple analysis, one gets that the Fourier mode $(\mathscr{F} \dot{\zeta}(k), \mathscr{F} \dot{v}(k))$ is stable (in the sense of $\S 3.2$ ) for the equations (36) if and only if

$g^{\prime}\left(1+\frac{\sigma}{g^{\prime}\left(\rho^{+}+\rho^{-}\right)} k^{2}\right)\left(1+\alpha H_{0}^{2} k^{2}\right)>\underline{\rho}^{+} \underline{\rho}^{-} \frac{1}{H_{0}} \llbracket c^{ \pm} \rrbracket^{2}\left(1+\frac{1}{3}\left(H^{+}\right)^{2} k^{2}\right)\left(1+\frac{1}{3}\left(H^{-}\right)^{2} k^{2}\right)$,

or equivalently

$$
\llbracket c^{ \pm} \rrbracket^{2}<\Omega_{K H} \alpha_{G N_{\sigma}}(k)
$$

with

$$
\alpha_{G N_{\sigma}}(k)=\left(1+\frac{1}{\mathrm{Bo}} H_{0}^{2} k^{2}\right) \alpha_{G N}(k)
$$

and

$$
\alpha_{G N}(k)=\frac{1}{H_{0}}\left(\frac{\underline{\rho}^{+} H^{-}}{\left(1+\frac{1}{3}\left(H^{-}\right)^{2} k^{2}\right)}+\frac{\underline{\rho}^{-} H^{+}}{\left(1+\frac{1}{3}\left(H^{+}\right)^{2} k^{2}\right)}\right) .
$$

It follows immediately that the modes with low wavenumber are stable if and only if

$$
(\text { Stab } 1)_{G N_{\sigma}} \quad \llbracket c^{ \pm} \rrbracket^{2}<\Omega_{K H},
$$

which is exactly the same as the condition (Stab 1) and (Stab 1) $)_{S W}$ derived respectively for the full equations and the SW/SW model. If it is satisfied, there is a unique $k_{G N_{\sigma}} \in \mathbb{R}_{*}^{+} \cup\{+\infty\}$ for each $\llbracket c^{ \pm} \rrbracket$, such that all the modes corresponding to $\xi \in \mathbb{R}^{d}$ with $|\xi|<k_{G N_{\sigma}}$ are stable,

$$
\text { (Critical wavenumber })_{G N_{\sigma}} \quad k_{G N_{\sigma}}=\min \alpha_{G N_{\sigma}}^{-1}\left(\frac{\left|\llbracket c^{ \pm} \rrbracket\right|^{2}}{\Omega_{K H}}\right) .
$$

In fact, one always has (at least for reasonable values of $\sigma$ as in Footnote 3) that $\alpha_{G N_{\sigma}}(k)<\alpha_{\sigma}(r)$ for $\sigma \geq 0$, with $\alpha_{\sigma}$ as in (26). Therefore we find $k_{G N_{\sigma}}<k_{K H}$ (see Figure 5(b)), and the GN/GN model (35) therefore overestimates Kelvin-Helmholtz instabilities (see Figure 5).

In absence of surface tension $(\sigma=0), \alpha_{G N_{\sigma}}(\cdot)=\alpha_{G N}(\cdot)$ is a decreasing function and goes to zero at infinity. As for the full equations, there are therefore always unstable modes in presence of a nonzero shear $\llbracket c^{ \pm} \rrbracket$, as noted in [13]. In presence of surface tension, the function $\alpha_{G N_{\sigma}}(\cdot)$ is still decaying ${ }^{3}$ but tends to a non zero limit at infinity. There exists therefore a critical value $\Omega_{G N_{\sigma}}^{c r}:=\Omega_{K H} \min _{\mathbb{R}^{+}} \alpha_{G N_{\sigma}}$ such that all modes are stable if and only if

$$
(\operatorname{Stab} 2)_{G N_{\sigma}} \quad \llbracket c^{ \pm} \rrbracket^{2} \leq \Omega_{G N_{\sigma}}^{c r}=\Omega_{K H} \frac{3}{\mathrm{Bo}}\left(1+\underline{\rho}^{+} \underline{\rho}^{-} \frac{\left(H^{+}-H^{-}\right)^{2}}{H^{+} H^{-}}\right) ;
$$

${ }^{3}$ If $\sigma$ is very large, $\alpha_{G N_{\sigma}}(\cdot)$ is no longer decreasing over $\mathbb{R}^{+}$. However, if $\sigma$ satisfies

$$
\sigma \leq \frac{1}{3} g^{\prime}\left(\rho^{+}+\rho^{-}\right) \min \left\{\left(H^{+}\right)^{2},\left(H^{-}\right)^{2}\right\},
$$

which is always satisfied in realistic physical configurations, then $\alpha_{G N_{\sigma}}(\cdot)$ is indeed a decreasing function. We always assume that we are in such a regime. 
the same analysis as for the discussion on the critical wave number shows that $\Omega_{G N_{\sigma}}^{c r}<\Omega^{c r}$; the GN/GN model being unable to reproduce correctly the threshold for Kelvin-Helmholtz instabilities, we are led to derive other models in the next sections. Note that contrary to the standard GN/GN model which has a Hamiltonian structure [15], these regularized models are in general not Hamiltonian (see Section 4 of [6] for a discussion on this aspect in the weakly nonlinear case).

\subsubsection{A first class of regularized models}

As shown in $\S 4.2 .3$, the second order shallow water model (or Green-Naghdi/GreenNaghdi) is always unstable for large wave numbers in absence of surface tension (even if the shear $\llbracket c^{ \pm} \rrbracket$ is very small). This led Choi, Barros and Jo [13] to derive an asymptotically equivalent model with better high frequency behavior in the sense that for a small enough shear $\llbracket c^{ \pm} \rrbracket$, all Fourier modes are stable.

Their idea was to use a technique commonly used in the one fluid case (water waves) to improve the dispersion relation of asymptotic models; it consists in working with a velocity variable that differs from the averaged velocity $\bar{v}$; this can typically be the velocity at some fixed depth or a given level line, of the fluid domain (see [39, 5, 7] and more generally Section 5.2 of [33]). This approach has also been used in the context of interfacial waves [8, 38]; the idea in [13] (see also [2], and [16] for a related approach) was to use it to remove the Kelvin-Helmholtz instabilities from the standard GN/GN model. The authors rewrote the GN/GN equations (35) in the variables $\left(\zeta, \hat{v}_{r}^{ \pm}\right)$instead of $\left(\zeta, \bar{v}^{ \pm}\right)$, where $\hat{v}_{r}^{ \pm}$is the horizontal velocity at the fixed height $\hat{z}_{r}^{ \pm}$, with

$$
\hat{z}_{r}^{ \pm}=\mp H^{ \pm}(1-\sqrt{-2 r+1 / 3}), \quad-1 / 3 \leq r \leq 1 / 6
$$

(so that $\hat{z}_{r}^{ \pm}=0$ if $r=-1 / 3$ and $\hat{z}_{r}^{ \pm}=\mp H^{ \pm}$if $r=1 / 6$ ). Using a standard shallow water expansion of the velocity field in the fluid domain (see for instance [41], $\S 13.11$ or [33], $\S 5.6 .2), \hat{v}_{r}^{ \pm}$can be related to $\bar{v}^{ \pm}$through the relation

$$
\bar{v}^{ \pm} \sim \hat{v}_{r}^{ \pm}-\frac{1}{6} H^{ \pm}(\zeta)^{2} \partial_{x}^{2} \hat{v}_{r}^{ \pm}+\left(-r+\frac{1}{6}\right)\left(H^{ \pm}\right)^{2} \partial_{x}^{2} \hat{v}_{r}^{ \pm} \quad\left(H^{ \pm}(\zeta)=H^{ \pm} \pm \zeta\right)
$$

The regularized equations of [13] are obtained by replacing $\bar{v}^{ \pm}$by this approximation in (35) and dropping smallest order terms. For $r=0$, the linearization of these equations around the rest state coincides with the linearization of the standard GN/GN model, but the nonlinear terms differ. Inspired by $\$ 5.2$ of [33] we therefore propose a slight modification (and generalization to the two dimensional case $d=2$ ) to the approach of [13] which consists in working with the velocity $V_{r}^{ \pm}$defined as

$$
V_{r}^{ \pm}=\left(1+3 r^{ \pm} \mathscr{T}^{ \pm}\right)^{-1} \bar{V}^{ \pm} \quad(r \geq 0) \quad\left(\text { with } r^{ \pm}=\frac{H_{0}^{2}}{\left(H^{ \pm}\right)^{2}} r \quad \text { and } r \geq 0\right),
$$

so that, with $\mathscr{T}^{ \pm}$as in (34), 


$$
\bar{V}^{ \pm}=V_{r}^{ \pm}+3 r^{ \pm} \mathscr{T}^{ \pm} V_{r}^{ \pm}
$$

Replacing $\bar{V}^{ \pm}$by this approximation in (35) and dropping the $O\left(\left(\mu^{ \pm}\right)^{2}\right)$ terms (with $\mu^{ \pm}=\left(H^{ \pm}\right)^{2} / L^{2}, L$ being the typical horizontal scale), one obtains the following family of regularized GN/GN models indexed by the parameter $r \geq 0$,

$$
\left\{\begin{array}{l}
\partial_{t} \zeta \pm \nabla \cdot\left[H^{ \pm}(\zeta)\left(1+3 r^{ \pm} \mathscr{T}^{ \pm}\right) V_{r}^{ \pm}\right]=0 \\
{\left[1+\left(1+3 r^{ \pm}\right) \mathscr{T}^{ \pm}\right] \partial_{t} V_{r}^{ \pm}+g \nabla \zeta+\frac{1}{2} \nabla\left|V_{r}^{ \pm}\right|^{2}+Q_{r}^{ \pm}=-\frac{1}{\rho^{ \pm}} \nabla \underline{P}^{ \pm}}
\end{array}\right.
$$

where

$$
Q_{r}^{ \pm}:=Q^{ \pm}\left(V_{r}^{ \pm}\right)+3 r^{ \pm} \nabla\left(V_{r}^{ \pm} \cdot \mathscr{T}^{ \pm} V_{r}^{ \pm}\right)+3 r^{ \pm}\left(\partial_{t} \mathscr{T}^{ \pm}\right) V_{r}^{ \pm},
$$

where $Q^{ \pm}(\cdot)$ as defined before in original GN/GN model and

$$
\begin{aligned}
\left(\partial_{t} \mathscr{T}^{ \pm}\right) V_{r}^{ \pm}= & -\frac{1}{3 H^{ \pm}(\zeta)^{2}} \nabla \cdot\left(H^{ \pm}(\zeta) V_{r}^{ \pm}\right) \nabla\left(H^{ \pm}(\zeta)^{3} \nabla \cdot V_{r}^{ \pm}\right) \\
& +\frac{1}{H^{ \pm}(\zeta)} \nabla\left(H^{ \pm}(\zeta)^{2} \nabla \cdot\left(H^{ \pm}(\zeta) V_{r}^{ \pm}\right)\left(\nabla \cdot V_{r}^{ \pm}\right)\right)
\end{aligned}
$$

In its one dimensional form $d=1$, this model is a small variant of the system (3.7)(3.8) of [13]; the first interest of working with (37) is that the standard GN/GN model (35) is a particular case (corresponding to $r=0$ ) of (37), while it does not belong to any of the regularized systems derived in [13]. The second and main advantage is that the definition of $v_{r}^{ \pm}$makes sense ${ }^{4}$ for all $r \geq 0$ while the definition of $\hat{v}_{r}$ in [13] requires that $r \leq 1 / 6$.

As for the previous examples, we now restrict to the case $d=1$ to investigate how this regularized model handles the Kelvin-Helmholtz instabilities. Linearizing around the constant shear $\left(\zeta, v_{r}^{ \pm}\right)=\left(0, c^{ \pm}\right)$, we get

$$
\left\{\begin{array}{l}
\partial_{t} \dot{\zeta}+c^{ \pm} \partial_{x} \dot{\zeta} \pm H^{ \pm}\left(1-r H_{0}^{2} \partial_{x}^{2}\right) \partial_{x} \dot{v}_{r}^{ \pm}=0 \\
\left(1-\frac{1}{3}\left(3 r H_{0}^{2}+\left(H^{ \pm}\right)^{2}\right) \partial_{x}^{2}\right)\left(\partial_{t} \dot{v}_{r}^{ \pm}+c^{ \pm} \partial_{x} \dot{v}_{r}^{ \pm}\right)+g \partial_{x} \dot{\zeta}=-\frac{1}{\rho^{ \pm}} \partial_{x} \underline{P}^{ \pm}
\end{array}\right.
$$

The formulas of Lemma 2 must now be replaced by

$\dot{v}_{r}^{+}=\frac{H^{-}}{H_{0}} \dot{v}_{r}-\frac{\rho^{-}}{H_{0}} \llbracket c^{ \pm} \rrbracket\left(1-r H_{0}^{2} \partial_{x}^{2}\right)^{-1} \dot{\zeta}, \quad \dot{v}_{r}^{-}=-\frac{H^{+}}{H_{0}} \dot{v}_{r}-\frac{\rho^{+}}{H_{0}} \llbracket c^{ \pm} \rrbracket\left(1-r H_{0}^{2} \partial_{x}^{2}\right)^{-1} \dot{\zeta}$

so that, proceeding as for (36), we are led (in absence of surface tension) to the system

\footnotetext{
${ }^{4}$ When $r \geq 1 / 6$, there is no obvious physical meaning for $v_{r}^{ \pm}$. For $0 \leq r \leq 1 / 6$, and for small amplitude waves $v_{r}^{ \pm}$is the horizontal velocity evaluated on the level line $\left\{z=\hat{z}_{r}^{ \pm}\left(1 \pm \frac{\zeta}{H^{ \pm}}\right)+\zeta\right\}$ (see [33], §5.6.2).
} 


$$
\left\{\begin{array}{l}
\partial_{t} \dot{\zeta}+c_{0} \partial_{x} \dot{\zeta}+\frac{H^{+} H^{-}}{H_{0}}\left(1-r H_{0}^{2} \partial_{x}^{2}\right) \partial_{x} \dot{v}_{r}=0, \\
\left(1-(\alpha+r) H_{0}^{2} \partial_{x}^{2}\right)\left(\partial_{t} \dot{v}_{r}+c_{0} \partial_{x} \dot{\dot{v}}_{r}\right)+\gamma \llbracket c^{ \pm} \rrbracket H_{0}^{2} \partial_{x}^{3} \dot{v}_{r} \\
\quad+\left(g^{\prime}-\frac{\underline{\rho}^{+} \underline{\rho}^{-}}{H_{0}} \llbracket c^{ \pm} \rrbracket^{2}\left(1-r H_{0}^{2} \partial_{x}^{2}\right)^{-1}\left(1-(\beta+r) H_{0}^{2} \partial_{x}^{2}\right)\right) \partial_{x} \dot{\zeta}=0
\end{array}\right.
$$

where $\dot{v}=\underline{\rho}^{+} \dot{v}^{+}-\underline{\rho}^{-} \dot{v}^{-}$. The Fourier mode $(\mathscr{F} \dot{\zeta}(k), \mathscr{F} \dot{v}(k))$ is therefore stable for the system $\overline{(38)}$ if and only if

$$
\left|\llbracket c^{ \pm} \rrbracket\right|^{2} \leq \Omega_{K H} \alpha_{G N_{r}}(\xi)
$$

where, for all $r \geq 0$,

$$
\alpha_{G N_{r}}(k)=\underline{\rho}^{+} \frac{H^{-}}{H_{0}} \frac{1+r H_{0}^{2} k^{2}}{1+\left(\frac{1}{3}\left(H^{-}\right)^{2}+r H_{0}^{2}\right) k^{2}}+\underline{\rho^{-}} \frac{H^{+}}{H_{0}} \frac{1+r H_{0}^{2} k^{2}}{1+\left(\frac{1}{3}\left(H^{+}\right)^{2}+r H_{0}^{2}\right) k^{2}} .
$$

Comparing $\alpha_{G N_{r}}$ with $\alpha_{G N}$ from the standard GN/GN model, one of course finds that $\alpha_{G N_{r}}(k)=\alpha_{G N}(k)$ when $r=0$, since the standard GN/GN model then exactly coincides with (37). Both $\alpha_{G N_{r}}$ and $\alpha_{G N}$ are decreasing functions but when $r>0$, $\alpha_{G N_{r}}(k)$ tends to a nonzero value as $k \rightarrow \infty$ while $\alpha_{G N}(k)$ tends to 0 as $k \rightarrow \infty$. It follows similarly as before that there exists stable modes for low wavenumber if and only if

$$
(\operatorname{Stab} 1)_{G N_{r}} \quad\left|\llbracket c^{ \pm} \rrbracket\right|^{2}<\Omega_{K H}
$$

With this condition, the critical wave number corresponding to $\llbracket c^{ \pm} \rrbracket$ is

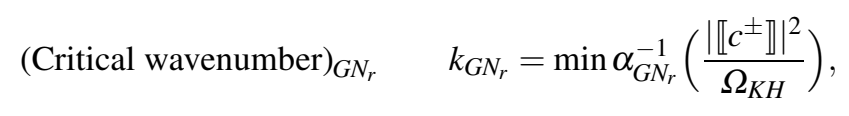

and there is a critical value $\Omega_{G N_{r}}^{c r}$ such that all modes are stable if and only if

$$
(\text { Stab 2) })_{G N_{r}} \quad \llbracket c^{ \pm} \rrbracket^{2} \leq \Omega_{G N_{r}}^{c r} ;
$$

one has $\Omega_{G N_{r}}^{c r}=\Omega_{K H} \min \alpha_{G N, r}$, that is

$$
\Omega_{G N_{r}}^{c r}=\Omega_{K H}\left[\underline{\rho}^{+} \frac{H^{-}}{H_{0}} \frac{r H_{0}^{2}}{\frac{1}{3}\left(H^{-}\right)^{2}+r H_{0}^{2}}+\underline{\rho}^{-} \frac{H^{+}}{H_{0}} \frac{r H_{0}^{2}}{\frac{1}{3}\left(H^{+}\right)^{2}+r H_{0}^{2}}\right] .
$$

In [13], the authors choose $r=1 / 6$, which gives the largest possible value for $\Omega_{G N_{r}}^{c r}$ (namely, $\left.\Omega_{G N_{r}}^{c r}=1 / 3 \Omega_{K H}\right)$ for a system written in $\left(\zeta, \hat{v}_{r}^{ \pm}\right)$and therefore subject to the constraint $r \geq 1 / 6$. Writing the regularized GN/GN model in terms of $\left(\zeta, V_{r}^{ \pm}\right)$as suggested here, it is possible to take any $r \geq 0$, and therefore $\Omega_{G N_{r}}^{c r}$ can take any value in $\left[0, \Omega_{K H}\right)$. The lower bound of this interval is achieved when $r=0$; the standard GN/GN is therefore the most unstable of the whole family of regularized models (37). The fact that the whole range $\left[0, \Omega_{K H}\right)$ can be covered with our approach implies in particular that it is possible to find some $r_{0} \geq 0$ such that the regularized model (37) 
has exactly the critical shear as the full equations (11)-(12) with surface tension, i.e. such that $\Omega_{G N_{r_{0}}}^{c r}=\Omega^{c r}$. One readily finds that

$$
r_{0}=\frac{-b+\sqrt{b^{2}-4 a c}}{2 a}
$$

with

$$
\begin{aligned}
& a=\left(1-\frac{\Omega_{c r}}{\Omega_{K H}}\right) H_{0}^{4}, \\
& b=\frac{H_{0}^{2}}{3}\left(H^{+} H^{-} \frac{\underline{\rho}^{+} H^{+}+\underline{\rho}^{-} H^{-}}{H_{0}}-\frac{\Omega_{c r}}{\Omega_{K H}}\left(\left(\left(H^{-}\right)^{2}+\left(H^{+}\right)^{2}\right)\right),\right. \\
& c=-\frac{1}{9} \frac{\Omega_{c r}}{\Omega_{K H}}\left(H^{+} H^{-}\right)^{2} .
\end{aligned}
$$

Remark 7. The lines above show that it is possible to reproduce the same behavior with respect to the apparition of Kelvin-Helmholtz instabilities as for the full Euler equations with surface tension, even though surface tension is not taken into account in (37). This is because the regularization used here can behave as a control mechanism for high frequencies that can be tuned to reproduce the surface tension effects in the full Euler equations. Taking surface tension into account in (37) would lead to a stability condition of the form

$$
\left|\llbracket c^{ \pm} \rrbracket\right|^{2} \leq \Omega_{K H} \alpha_{G N_{\sigma, r}}(k) \quad \text { with } \quad \alpha_{G N_{\sigma, r}}(k)=\left(1+\frac{1}{\mathrm{Bo}} H_{0}^{2} k^{2}\right) \alpha_{G N_{r}}(k) .
$$

\subsubsection{A second class of regularized models}

In the previous section, we extended the approach of [13] and obtained a very flexible approach to describe the formation of Kelvin-Helmholtz instabilities. There is however a drawback when working with the models of [13] and more generally with the regularized models (37). Indeed, the first equation in (35) is exact (conservation of mass), while the first equation in (37) is only valid up to order $O\left(\left(\mu^{ \pm}\right)^{2}\right)$ when $r \neq 0$ (we recall that $\mu^{ \pm}=\left(H^{ \pm}\right)^{2} / L^{2}$ where $L$ is the typical horizontal scale).

We propose here a second family of regularized models, that keeps the same advantages as (37) to handle Kelvin-Helmholtz instabilities, but for which the conservation of mass is also exact.

We recall that at first order in the shallowness parameter $\mu^{ \pm}$, the full equations (11) can be approximated by the SW/SW equations (29) and that the GN/GN equations (35) are a second order approximation. In particular, the second equation of (35) can be written under the form

$$
\partial_{t} \bar{V}^{ \pm}=-g \nabla \zeta-\frac{1}{2} \nabla\left|\bar{V}^{ \pm}\right|^{2}-\frac{1}{\rho^{ \pm}} \nabla \underline{P}^{ \pm}+O\left(\mu^{ \pm}\right) .
$$


We now use a generalization of the so called BBM trick, namely, we write

$$
\begin{gathered}
\mathscr{T}^{ \pm} \partial_{t} \bar{V}^{ \pm}=\left(1+3 s^{ \pm}\right) \mathscr{T}^{ \pm} \partial_{t} \bar{V}^{ \pm}+3 s^{ \pm} g \mathscr{T}^{ \pm} \nabla \zeta+3 s^{ \pm} \frac{1}{2} \mathscr{T}^{ \pm} \nabla\left|\bar{V}^{ \pm}\right|^{2} \\
+3 s^{ \pm} \frac{1}{\rho^{ \pm}} \mathscr{T}^{ \pm} \nabla \underline{P}^{ \pm}+O\left(\mu^{ \pm}\right)
\end{gathered}
$$

(with $s^{ \pm}=\frac{H_{0}^{2}}{\left(H^{ \pm}\right)^{2}} s$ where $s \in \mathbb{R}$ ), and plug this into the second equation of (35) to obtain the following family of regularized GN/GN systems indexed by the parameters $s^{ \pm}$as

$$
\left\{\begin{array}{l}
\partial_{t} \zeta \pm \nabla \cdot\left(H^{ \pm}(\zeta) \bar{V}^{ \pm}\right)=0, \\
\left(1+\left(1+3 s^{ \pm}\right) \mathscr{T}^{ \pm}\right) \partial_{t} \bar{V}^{ \pm}+g \nabla \zeta+\frac{1}{2} \nabla\left|\bar{V}^{ \pm}\right|^{2}+Q_{s}^{ \pm}=-\frac{1}{\rho^{ \pm}}\left(1+3 s^{ \pm} \mathscr{T}^{ \pm}\right) \nabla \underline{P}^{ \pm}
\end{array}\right.
$$

with

$$
Q_{s}^{ \pm}=Q^{ \pm}+3 s^{ \pm}\left(g \mathscr{T}^{ \pm} \nabla \zeta+\frac{1}{2} \mathscr{T}^{ \pm} \nabla\left|\bar{V}^{ \pm}\right|^{2}\right) .
$$

Focusing on the 1-D case and linearizing this system around the constant shear $\left(c^{+}, c^{-}\right)$, one finds the following linear system

$$
\left\{\begin{array}{l}
\partial_{t} \dot{\zeta}+c^{ \pm} \partial_{x} \dot{\zeta} \pm H^{ \pm} \partial_{x} \dot{v}^{ \pm}=0 \\
\left(1-\left(\frac{1}{3}+s^{ \pm}\right)\left(H^{ \pm}\right)^{2} \partial_{x}^{2}\right)\left(\partial_{t} \dot{v}^{ \pm}+c^{ \pm} \partial_{x} \dot{v}^{ \pm}\right)+g\left(1-s^{ \pm}\left(H^{ \pm}\right)^{2} \partial_{x}^{2}\right) \partial_{x} \dot{\zeta} \\
\quad=-\frac{1}{\rho^{ \pm}}\left(1-s^{ \pm}\left(H^{ \pm}\right)^{2} \partial_{x}^{2}\right) \nabla \underline{P}^{ \pm}
\end{array}\right.
$$

Using Lemma 2 again to express $\dot{v}^{ \pm}$in terms of $\dot{v}=\underline{\rho}^{+} \dot{v}^{+}-\underline{\rho}^{-} \dot{v}^{-}$and $\dot{\zeta}$, and proceeding as for (36), this system can be rewritten as

$$
\left\{\begin{array}{l}
\partial_{t} \dot{\zeta}+c_{0} \partial_{x} \dot{\zeta}+\frac{H^{+} H^{-}}{H_{0}} \partial_{x} \dot{v}=0 \\
\left(1-(\alpha+s) H_{0}^{2} \partial_{x}^{2}\right)\left(\partial_{t} \dot{v}+c_{0} \partial_{x} \dot{v}\right)+\gamma \llbracket c^{ \pm} \rrbracket H_{0}^{2} \partial_{x}^{3} \dot{v} \\
\quad+\left(g^{\prime}\left(1-s H_{0}^{2} \partial_{x}^{2}\right)-\frac{\underline{\rho}^{+} \underline{\rho}^{-}}{H_{0}} \llbracket c^{ \pm} \rrbracket^{2}\left(1-(\beta+s) H_{0}^{2} \partial_{x}^{2}\right)\right) \partial_{x} \dot{\zeta}=0,
\end{array}\right.
$$

where $\alpha, \beta, \gamma$ and $c_{0}$ are the same as before.

With a simple analysis, one gets that the Fourier mode $(\mathscr{F} \dot{\zeta}(k), \mathscr{F} \dot{v}(k))$ is stable (in the sense of §3.2) for the equations (41) if and only if

$$
\llbracket c^{ \pm} \rrbracket^{2}<\Omega_{K H} \alpha_{G N_{s}}(k)
$$

with

$$
\alpha_{G N_{s}}(k)=\underline{\rho}^{+} \frac{H^{-}}{H_{0}} \frac{1+s H_{0}^{2} k^{2}}{1+\left(\frac{1}{3}\left(H^{-}\right)^{2}+s H_{0}^{2}\right) k^{2}}+\underline{\rho}^{-} \frac{H^{+}}{H_{0}} \frac{1+s H_{0}^{2} k^{2}}{1+\left(\frac{1}{3}\left(H^{+}\right)^{2}+s H_{0}^{2}\right) k^{2}} ;
$$


this is exactly the same as the function $\alpha_{G N_{r}}$ derived for the first regularization (37). Consequently, keeping an exact equation for the conservation of mass, the second regularization (40), provides the same description of the formation of KelvinHelmholtz instabilities as (37).

\subsection{Application}

We apply here some of the results derived above to analyze some experimental data that can be found in [29], where the propagation of interfacial solitary waves is studied; it is in particular shown that large amplitude solitary waves are destroyed by Kelvin-Helmholtz instabilities.

First of all, in the case of solitary waves, one can express the shear speed $\llbracket c^{ \pm} \rrbracket$ with the wave speed $c$ and the amplitude of solitary wave $a$. In fact, plugging the transverse solution $\zeta(x-c t)$ into the first equation of (35) and integrating with respect to $x$ in $1-\mathrm{D}$ case results in

$$
-c \zeta \pm H^{ \pm}(\zeta) \bar{v}^{ \pm}=0
$$

where we assume $\zeta, \bar{v} \rightarrow 0$ when $x$ goes to $\infty$. This implies that at the bottom point of the solitary wave, i.e. $\zeta=a$ (with $a<0$ for a depression solitary wave), one has

$$
\bar{v}^{ \pm}= \pm \frac{c a}{H^{ \pm} \mp a} .
$$

If we define the jump of speed $\llbracket c^{ \pm} \rrbracket$ as $\llbracket c^{ \pm} \rrbracket:=\bar{v}^{+}-\bar{v}^{-}$, we can have

$$
\llbracket c^{ \pm} \rrbracket=\frac{c a\left(H^{+}+H^{-}\right)}{\left(H^{+}-a\right)\left(H^{-}+a\right)} .
$$

One can also find this formula in [13].

Now we want to fix the value of surface tension coefficient $\sigma$ for the linearized full system (22) according to this expression, the critical value $\Omega^{c r}$ for (22) and the experimental data in [29].

One has from [29] that $\rho^{+}=1022 \mathrm{~kg} / \mathrm{m}^{3}, \rho^{-}=999 \mathrm{~kg} / \mathrm{m}^{3}$ (hence $\rho^{+}=0.506$, $\left.\underline{\rho}^{-}=0.494\right), H^{+}=0.62 \mathrm{~m}$ and $H^{-}=0.15 \mathrm{~m}$. Taking the experimental values $\bar{c} / c_{0}-1=0.24$ and $a / H^{-}=1.23$ (corresponding to the last experiment before the apparition of Kelvin-Helmholtz instabilities in Figure 7 in [29]), where $c_{0}^{2}=$ $\frac{g^{\prime} H^{+} H^{-}}{\rho^{+} H^{-}+\rho^{+} H^{+}}$is the linear long wave speed from $\mathrm{KdV}$ equation, one can compute that $\llbracket c^{ \pm} \rrbracket=0.20 \mathrm{~m} / \mathrm{s}$ at the onset of Kelvin-Helmholtz instabilities. This means that, with the notations of $\S 3.2$, one has $\Omega^{c r}=\llbracket c^{ \pm} \rrbracket^{2}=0.04(\mathrm{~m} / \mathrm{s})^{2}$. Using Proposition 1, we use the approximation $\Omega_{+}^{c r} \sim \Omega^{c r}$ and the definition of $\Omega_{+}^{c r}$ to get

$$
\frac{1}{\mathrm{Bo}}=\frac{1}{4}\left(\frac{\Omega_{+}^{c r}}{\Omega_{K H}}\right)^{2} \sim \frac{1}{4}\left(\frac{0.04}{\Omega_{K H}}\right)^{2} ;
$$


from the definition (5) of the Bond number, we deduce that

$$
\sigma \sim \frac{1}{4 g^{\prime}}(0.04)^{2} \frac{\left(\rho^{+} \rho^{-}\right)^{2}}{\left(\rho^{+}+\rho^{-}\right)^{3}}=0.45
$$

(using this value of $\sigma$, on can compute that the lower bound estimate for $\Omega_{c r}$ provided by Proposition 1 is $\Omega_{c r}^{-}=0.038$ so that the error made by approximating $\Omega_{c r}$ by $\Omega_{c r}^{+}$to compute $\sigma$ is only $3 \%$ ). This value of $\sigma$ is significantly larger than the air-water surface tension $(\sigma \sim 0.07)$, which was to be expected because we treat here the surface tension as a model to describe the various different stabilizing effects (such as the mixing layer) between two miscible fluids. Note however that even though $\sigma$ is larger than usual, it does does not significantly affect the behavior of the wave while Kelvin-Helmholtz instabilities do not destroy it. Indeed, surface tension in (36) plays a role through a term of the form $-\sigma /\left(\rho^{+}+\rho^{-}\right) \partial_{x}^{3} \zeta$ while the dispersive term coming from non-hydrostatic terms is $-\alpha H_{0}^{2} \partial_{x}^{2} \partial_{t} v \sim \alpha g^{\prime} H_{0}^{2} \partial_{x}^{3} \zeta$. Using the above data then shows that the dispersive effects coming from surface tension are only about $5.9 \%$ of non hydrostatic ones. This confirms the general picture exhibited in [32], namely, that surface tension is necessary for the wave to exist, but that it does not affect significantly its propagation.

As shown in $\$ 4.2 .4$, it is also possible to find some $r_{0}$ such that the regularized model (37) reproduces the same critical value for the apparition of KelvinHelmholtz instabilities (i.e. let $\Omega_{G N_{r}}^{c r}=\Omega^{c r}$ ); according to (39), one gets $r_{0}=0.12$. Note in particular that $0<r_{0}<1 / 6$ and is therefore in the range allowed by the regularized models of [13], but close to its upper range. Slightly more stable configurations would therefore fall out of the range of validity of [13], but would stay within the range allowed for (37); Since the stability criterion for the second family of regularized systems (40) is the same as for (37), these comments hold for these models also.

\section{The Kelvin-Helmholtz instability in the free surface case}

We investigate here the formation of (linear) Kelvin-Helmholtz instabilities for twofluids interfaces in the case of a free top surface. In fact, we consider again the linear stability of perturbations near a constant shear flow as we did in the rigid lid case, and we follow the formulation above.

With a free top surface, we get a more complex system of $\left(\zeta_{s}, U_{\|}^{s}, \zeta, U_{\|}^{ \pm}\right)$(see $\S 2.2$ ), and the linear stability analysis becomes more difficult. We perform some numerical computations, and are able to find the expressions of the foot points for the stable area in some special cases. A comparison with the rigid lid case is also done in this section, where we discuss in particular the presence of a new range of stable frequencies. 


\subsection{Linearization around a constant shear $\left(\mathrm{c}^{+}, \mathrm{c}^{-}\right)$}

We consider here the linear equations governing small perturbations of the constant horizontal shear $\zeta^{s}=\zeta=0, V^{ \pm}=\mathbf{c}^{ \pm}, w^{ \pm}=0$ (and therefore $U_{\|}^{ \pm}=\mathbf{c}^{ \pm}, U_{\|}^{s}=\mathbf{c}^{-}$). Linearizing (17)-(19) around $\zeta^{s}=\zeta=0, \underline{V}^{s}=\mathbf{c}^{-}, \underline{V}^{ \pm}=\mathbf{c}^{ \pm}$we find the following linear equations for the perturbation $\left(\dot{\zeta}^{s}, \dot{V}^{s}, \dot{\zeta}, \dot{V}^{ \pm}\right)$

$$
\left\{\begin{array}{l}
\partial_{t} \dot{\zeta}^{s}+\mathbf{c}^{-} \cdot \nabla \dot{\zeta} s-\dot{w}^{s}=0 \\
\partial_{t} \dot{V}^{s}+g \nabla \dot{\zeta} s+\mathbf{c}^{-} \cdot \nabla \dot{V}^{s}=0 \\
\partial_{t} \dot{\zeta}+\mathbf{c}^{ \pm} \cdot \nabla \dot{\zeta}-\dot{w}^{ \pm}=0 \\
\partial_{t} \dot{V}^{ \pm}+g \nabla \dot{\zeta}+\mathbf{c}^{ \pm} \cdot \nabla \dot{V}^{ \pm}=-\frac{1}{\rho^{ \pm}} \nabla \underline{P}^{ \pm}
\end{array}\right.
$$

(we refer to Lemma 3 for an expression of $\dot{w}^{ \pm}$), with

$$
\llbracket \underline{P}^{ \pm} \rrbracket=-\sigma \Delta \dot{\zeta}
$$

As for (21), this can be further reduced by introducing $\dot{V}=\underline{\rho}^{+} \dot{V}^{+}-\underline{\rho}^{-} \dot{V}^{-}$; one gets the following free surface version of (21)

$$
\left\{\begin{array}{l}
\partial_{t} \dot{\zeta}^{s}+\mathbf{c}^{-} \cdot \nabla \dot{\zeta} s-\dot{w}^{s}=0 \\
\partial_{t} \dot{V}^{s}+g \nabla \dot{\zeta}+\mathbf{c}^{-} \cdot \nabla \dot{V}^{s}=0 \\
\partial_{t} \dot{\zeta}+\mathbf{c}^{ \pm} \cdot \nabla \dot{\zeta}-\dot{w}^{ \pm}=0 \\
\partial_{t} \dot{V}+\left\langle\mathbf{c}^{ \pm}\right\rangle \cdot \nabla \dot{V}+g^{\prime} \nabla \dot{\zeta}+\llbracket \mathbf{c}^{ \pm} \rrbracket \cdot \nabla\left\langle\underline{\rho}^{ \pm} \dot{V}^{ \pm}\right\rangle=\frac{\sigma}{\rho^{+}+\rho^{-}} \nabla \Delta \dot{\zeta} .
\end{array}\right.
$$

We then need the following lemma (similar to Lemma 1) to handle the free surface case.

Lemma 3. One has

$$
\begin{aligned}
& \dot{V}^{+}=\frac{\underline{\rho}^{-}}{b(D)}\left(\llbracket \mathbf{c}^{ \pm} \rrbracket \cdot \nabla\right) \nabla \dot{\zeta}+\frac{\mathrm{cth}^{-}|D|}{b(D)} \dot{V}+\frac{\underline{\rho}^{-}|D|}{\operatorname{sh}^{-} b(D)} \dot{V}^{s} \\
& \dot{V}^{-}=\frac{\underline{\rho}^{+}}{b(D)}\left(\llbracket \mathbf{c}^{ \pm} \rrbracket \cdot \nabla\right) \nabla \dot{\zeta}-\frac{\operatorname{th}^{+}|D|}{b(D)} \dot{V}+\frac{\underline{\rho}^{+}|D|}{\operatorname{sh}^{-} b(D)} \dot{V}^{s}
\end{aligned}
$$

and

$$
\dot{w}^{+}=-\mathrm{th}^{+} \frac{\nabla}{|D|} \cdot \dot{V}^{+}, \quad \dot{w}^{-}=\mathrm{cth}^{-} \frac{\nabla}{|D|} \cdot \dot{V}^{-}-\frac{1}{\mathrm{sh}^{-}} \frac{\nabla}{|D|} \cdot \dot{V}^{s}
$$

and

$$
\dot{w}^{s}=\frac{1}{\operatorname{sh}^{-}} \frac{\nabla}{|D|} \cdot \dot{V}^{-}-\mathrm{cth}^{-} \frac{\nabla}{|D|} \cdot \dot{V}^{s}
$$

with the notation $b(D)=\left(\underline{\rho}^{+} \mathrm{cth}^{-}+\underline{\rho}^{-} \mathrm{th}^{+}\right)|D|$.

Proof. As in the proof of Lemma 1, we write $\dot{V}^{ \pm}=\nabla \dot{\psi}^{ \pm}, \dot{V}=\nabla \dot{\psi}$ with $\dot{\psi}=$ $\underline{\rho}^{+} \dot{\psi}^{+}-\underline{\rho}^{-} \dot{\psi}^{-}$, we also write $\dot{V}^{s}=\nabla \dot{\psi}^{s}$. 
Let $\dot{\phi}^{-}$be the harmonic potential in the upper strip $0<z<H^{-}$and such that $\dot{\phi}_{\left.\right|_{z=0} ^{-}}^{-}=\dot{\psi}^{-}$and $\dot{\phi}_{\left.\right|_{z=H^{-}} ^{-}}^{-}=\dot{\psi}^{s}$. Explicit computations show that

$$
\dot{\phi}^{-}=\frac{\sinh (z|D|)}{\sinh \left(H^{-}|D|\right)} \dot{\psi}^{s}-\frac{\sinh \left(\left(z-H^{-}\right)|D|\right)}{\sinh \left(H^{-}|D|\right)} \dot{\psi}^{-},
$$

from which we deduce that

$$
\underline{w}^{-}=\partial_{z} \dot{\phi}_{\left.\right|_{z=0} ^{-}}=\frac{1}{\mathrm{sh}^{-}}|D| \dot{\psi}^{s}-\mathrm{cth}^{-}|D| \dot{\psi}^{-}
$$

Proceeding as in the proof of Lemma 1, we are therefore led to solve

$$
\begin{cases}|D| \mathrm{th}^{+} \dot{\psi}^{+}+|D| \mathrm{cth}^{-} \dot{\psi}^{-} & =\llbracket \mathbf{c}^{ \pm} \rrbracket \cdot \nabla \dot{\zeta}+\frac{1}{\mathrm{sh}^{-}}|D| \dot{\psi}^{s} \\ \underline{\rho}^{+} \dot{\psi}^{+}-\underline{\rho}^{-} \dot{\psi}^{-} & =\dot{\psi}\end{cases}
$$

this leads to

$$
\begin{aligned}
& \dot{\psi}^{+}=\frac{\underline{\rho}^{-}}{b(D)} \llbracket \mathbf{c}^{ \pm} \rrbracket \cdot \nabla \dot{\zeta}+\frac{\mathrm{cth}^{-}|D|}{b(D)} \dot{\psi}+\frac{\underline{\rho}^{-}|D|}{\mathrm{sh}^{-} b(D)} \dot{\psi}^{s} \\
& \dot{\psi}^{-}=\frac{\underline{\rho}^{+}}{b(D)} \llbracket \mathbf{c}^{ \pm} \rrbracket \cdot \nabla \dot{\zeta}-\frac{\mathrm{th}^{+}|D|}{b(D)} \dot{\psi}+\frac{\underline{\rho}^{+}|D|}{\mathrm{sh}^{-} b(D)} \dot{\psi}^{s}
\end{aligned}
$$

and the formulas of the lemma follow easily after remarking that

$$
\dot{w}^{s}=\mathrm{cth}^{-}|D| \psi^{s}-\frac{|D|}{\mathrm{sh}^{-}} \dot{\psi}^{-} .
$$

This ends the proof.

Thanks to the lemma, we can rewrite (42) under the form

$$
\partial_{t} W+A(D) W=0
$$

where $W=\left(\dot{\zeta}^{s}, \dot{V}^{s}, \dot{\zeta}, \dot{V}\right)$ and

$$
A(D)=\left(\begin{array}{cccc}
\mathbf{c}^{-} \cdot \nabla & \frac{b_{0}(D) \nabla}{b(D) \mathrm{th}^{-}} . & \frac{\underline{\rho}^{+}|D| \llbracket \mathbf{c}^{ \pm} \rrbracket \cdot \nabla}{b(D) \mathrm{sh}^{-}} & \frac{\mathrm{th}^{+} \nabla}{b(D) \mathrm{sh}^{-}} \\
g \nabla & \mathbf{c}^{-} \cdot \nabla & 0 & 0 \\
0 & \frac{\underline{\rho}^{-} \mathrm{th}^{+} \nabla}{b(D) \mathrm{sh}^{-}} & \overline{\mathbf{c}}(D) \cdot \nabla & \frac{\mathrm{th}^{+} \mathrm{cth}^{-} \nabla}{b(D)} \cdot \\
0 & \frac{\underline{\underline{\rho}}^{+} \underline{\underline{\rho}}|-D|}{b(D) \mathrm{sh}^{-}}\left(\llbracket \mathbf{c}^{ \pm} \rrbracket \cdot \nabla\right) & a(D) \nabla & \overline{\mathbf{c}}(D) \cdot \nabla
\end{array}\right)
$$

where $b(D)=\left(\underline{\rho}^{+} \mathrm{cth}^{-}+\underline{\rho}^{-} \mathrm{th}^{+}\right)|D|$ is already defined in Lemma $3, b_{0}(D)=$ $\underline{\rho}^{+} \mathrm{th}^{-}+\underline{\rho}^{-} \mathrm{th}^{+}$, and moreover 
$\overline{\mathbf{c}}(D)=\frac{\left(\mathbf{c}^{+} \underline{\rho}^{+} \mathrm{cth}^{-}+\mathbf{c}^{-} \underline{\rho}^{-} \mathrm{th}^{+}\right)|D|}{b(D)}, \quad a(D)=g^{\prime}+\frac{\underline{\rho}^{+} \underline{\rho}^{-}}{b(D)}\left(\llbracket c^{ \pm} \rrbracket \cdot \nabla\right)^{2}-\frac{\sigma}{\rho^{+}+\rho^{-}} \Delta$.

\subsection{Kelvin-Helmholtz instabilities for the linearized two-fluids equations with free surface}

In this section we only focus on the $d=1$ case for the sake of clarity. As in $\S 3.2$, we write $\llbracket \mathbf{c}^{ \pm} \rrbracket=\llbracket c^{ \pm} \rrbracket \mathbf{e}_{x}$ and $\mathbf{k}=k \mathbf{e}_{x}$, with $\llbracket c^{ \pm} \rrbracket=\left|\llbracket \mathbf{c}^{ \pm} \rrbracket\right|$ and $k=|\mathbf{k}|$.

In order to find out the stability criterion for the linearized system (43), we need to explore the eigenvalue $\omega$ for the Fourier mode $A(k)$ of operator $A(D)$. Indeed, $\omega$ satisfies the equation

$$
a_{0} x^{4}+a_{1} x^{3}+a_{2} x^{2}+a_{3} x+a_{4}=0
$$

where $x=c^{-}-\frac{\omega}{i k}$ and

$$
\begin{aligned}
& a_{0}=1, \quad a_{1}=\frac{2 \underline{\rho}^{+} \mathrm{cth}^{-} k \llbracket c^{ \pm} \rrbracket}{b(k)} \\
& a_{2}=\frac{\mathrm{cth}^{-}}{b(k)}\left(\underline{\rho}^{+} k \llbracket c^{ \pm} \rrbracket^{2}-g b_{0}(k)-\left(g^{\prime}+\frac{\sigma}{\rho^{+}+\rho^{-}} k^{2}\right) \mathrm{th}^{+}\right), \\
& a_{3}=-\frac{2 g \underline{\rho}^{+} \llbracket c^{ \pm} \rrbracket}{b(k)}, \quad a_{4}=-\frac{g \underline{\rho}^{+} \llbracket c^{ \pm} \rrbracket^{2}}{b(k)}+\frac{g \operatorname{th}^{+}}{b(k) k}\left(g^{\prime}+\frac{\sigma}{\rho^{+}+\rho^{-}} k^{2}\right),
\end{aligned}
$$

and we write $b=b(k)$ and $b_{0}=b_{0}(k)$ for short in the following text. The linear system (43) is stable if and only if all the eigenvalues $\omega$ are pure imaginary, that is, all the roots $x$ for the equation above are real. As in [1], we use [28] to express the stability criterion under the form

$$
\Delta_{3}>0, \Delta_{5}>0, \Delta_{7}>0 \quad \text { or } \quad \Delta_{3} \geq 0, \Delta_{5}=0, \Delta_{7}=0
$$

where

$$
\Delta_{7}=\left|\begin{array}{ccccccc}
a_{0} & a_{1} & a_{2} & a_{3} & a_{4} & 0 & 0 \\
0 & a_{0} & a_{1} & a_{2} & a_{3} & a_{4} & 0 \\
0 & 0 & a_{0} & a_{1} & a_{2} & a_{3} & a_{4} \\
0 & 0 & 0 & 4 a_{0} & 3 a_{1} & 2 a_{2} & a_{3} \\
0 & 0 & 4 a_{0} & 3 a_{1} & 2 a_{2} & a_{3} & 0 \\
0 & 4 a_{0} & 3 a_{1} & 2 a_{2} & a_{3} & 0 & 0 \\
4 a_{0} & 3 a_{1} & 2 a_{2} & a_{3} & 0 & 0 & 0
\end{array}\right|
$$

and $\Delta_{3}, \Delta_{5}$ are the $3 \times 3,5 \times 5$ inner center determinants.

Since these conditions are far more complicated than the stability criterion for the rigid lid case, we cannot write down the exact stability expressions and we prefer to use numerical computations. 
Compared to the stable area for the rigid lid case in section 3.2, Fig 6 tells us that there are two parts for the stable area of system (43). The left part is similar and close to the stable area for the rigid lid case, while there is a new stable part in the free surface case on the right-hand side. This means that the linear system can also be stable for high shear and low frequency, which is completely different from the rigid lid case.

When surface tension is neglected, i.e. $\sigma=0$, there are also two parts of stable area for the system (43). Similarly as the surface tension case above, the left part is also close to the stable area of the corresponding rigid lid case. In fact, the stable area is pretty much the same as the surface tension case in the low frequency part.

Summing up the numerical analysis, the stability condition for system (43) can be described roughly as

$$
\left|\llbracket c^{ \pm} \rrbracket\right|^{2} \leq \Omega_{K H, 1}(k) \quad \text { or } \quad \Omega_{K H, 2}(k) \leq\left|\llbracket c^{ \pm} \rrbracket\right|^{2} \leq \Omega_{K H, 3}(k)
$$

where $\Omega_{K H, i}(k)(i=1,2,3)$ can be expressed in terms of $k$, and $\Omega_{K H, 1}(k)$ is close to $\Omega_{K H} \alpha_{\sigma}(k)$ from the rigid lid case. So the modes with low wave number are stable if and only if

$$
\text { (Stab 1) } \quad\left|\llbracket c^{ \pm} \rrbracket\right|^{2} \leq \Omega_{K H, 1}(0) \quad \text { or } \quad\left|\llbracket c^{ \pm} \rrbracket\right|^{2} \geq \Omega_{K H, 2}(0) .
$$

We shall provide in $\S 6.1 .3$ exact values of $\Omega_{K H, 1}(0)$ and $\Omega_{K H, 2}(0)$ that will allow us to show another striking difference with the rigid lid case: one has $\Omega_{K H, 1}(0) \neq \Omega_{K H}$ (in fact $\Omega_{K H, 1}(0)<\Omega_{K H}$ in our numerical computations), that is, the threshold for the stability of small wavenumbers is not the same as in the rigid lid model (even if we restrict it to its left part corresponding to small shears. One can have the condition (Stab 2) for the stability of all wave numbers:

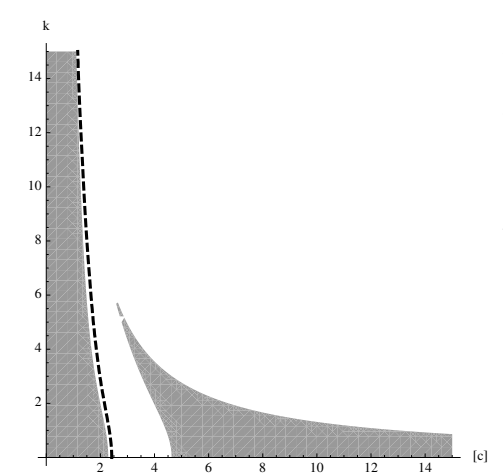

(a) the two parts of the stable area.

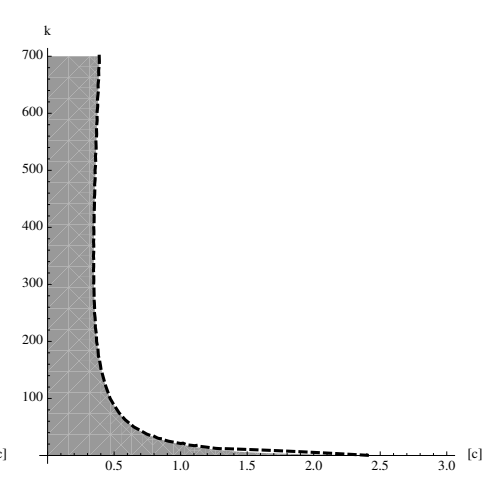

(b) a closer look at the left part.

Fig. 6 The stable area for the linear system (43) in free surface case with surface tension on the interface. The dashed line represents the bound for the stable area in the corresponding rigid lid case. Parameters $H^{+}=0.62, \delta=0.15 / 0.62, \rho^{+}+\rho^{-}=2021, \gamma=0.4, \sigma=0.073$ and $g=9.81$. 


$$
\left(\text { Stab 2) } \quad\left|\llbracket c^{ \pm} \rrbracket\right|^{2} \leq \Omega_{F S}^{c r}:=\min _{\mathbb{R}^{+}} \Omega_{K H, 1}(k)>0,\right.
$$

where we know from the numeral computations that $\Omega_{F S}^{c r}$ is close to the one $\Omega^{c r}$ in the corresponding rigid lid case and therefore can be estimated approximately by Prop 1.

Remark 8. In absence of surface tension, i.e. $\sigma=0$, we obtain that the stability condition for all wave numbers holds only for a zero shear, and there is therefore no difference with the rigid lid case as far as the stability condition (Stab 2) is concerned.

\section{Shallow water approximations in the free surface case}

This section investigates the (linear) Kelvin-Helmholtz instabilities of the shallow water approximation when the upper bound is a free surface. We consider here only a first-order approximation leading to a free surface version of the SW/SW system, where we are able to derive the exact expressions for stability criterion in the case when the two layers have the same average depths $\left(H^{+}=H^{-}\right)$. Based on this observation, we can understand more about the differences between the rigid lid case and the free surface case.

Notation: We need to adapt the definition of $\bar{V}^{ \pm}$in $\S 4$ to the free surface case as follows

$$
\begin{array}{r}
\bar{V}^{+}=\frac{1}{H^{+}(\zeta)} \int_{-H^{+}}^{\zeta} V^{+}(t, X, z) d z, \\
\bar{V}^{-}=\frac{1}{H^{-}\left(\zeta^{s}, \zeta\right)} \int_{\zeta}^{H^{-}+\zeta^{s}} V^{-}(t, X, z) d z, \\
\text { where } H^{+}(\zeta)=H^{+}+\zeta \text { and } H^{-}\left(\zeta^{s}, \zeta\right)=H^{-}+\zeta^{s}-\zeta .
\end{array}
$$

\subsection{First order approximation}

A first order approximation is discussed here in this section, similarly as in the SW/SW part in the rigid lid case. We linearize the system around a constant shear as before, and study numerically its stability; we also derive an explicit criterion in the particular case where both fluid layers have the same depth at rest.

\subsubsection{The Shallow Water/Shallow Water equations with free surface}

At first order, one writes 


$$
U_{\|}^{s} \sim \bar{V}^{-}, \quad U_{\|}^{ \pm} \sim \bar{V}^{ \pm}, \quad \text { and } \quad \underline{w}^{s} \sim 0, \quad \underline{w}^{ \pm} \sim 0 .
$$

Plugging this into system (17)-(18) one has the nonlinear SW/SW system as

$$
\left\{\begin{array}{l}
\partial_{t} \zeta^{s}+\nabla \cdot\left(H^{-}\left(\zeta^{s}, \zeta\right) \bar{V}^{-}\right)+\nabla \cdot\left(H^{+}(\zeta) \bar{V}^{+}\right)=0 \\
\partial_{t} \bar{V}^{-}+g \nabla \zeta^{s}+\frac{1}{2} \nabla|\bar{V}|^{2}=0 \\
\partial_{t} \zeta+\nabla \cdot\left(H^{+}(\zeta) \bar{V}^{+}\right)=0 \\
\partial_{t} \bar{V}^{ \pm}+g \nabla \zeta+\frac{1}{2} \nabla\left|\bar{V}^{ \pm}\right|^{2}=-\left.\frac{1}{\rho^{ \pm}} \nabla \underline{P}^{ \pm}\right|_{z=\zeta}
\end{array}\right.
$$

this system has been derived and studied in [14, 19, 3], and justified in [24].

\subsubsection{Linearization around a constant shear $\left(\mathrm{c}^{+}, \mathrm{c}^{-}\right)$}

For the sake of clarity, we only consider the 1-D case here, so we write $\mathbf{c}^{ \pm}$to be $c^{ \pm}$. After linearizing around the constant shear as before one obtains

$$
\left\{\begin{array}{l}
\partial_{t} \zeta^{s}+c^{-} \partial_{x} \zeta^{s}+H^{-} \partial_{x} \dot{v}^{-}+\llbracket c^{ \pm} \rrbracket \partial_{x} \zeta+H^{+} \partial_{x} \dot{v}^{+}=0, \\
\partial_{t} \dot{v}^{-}+g \partial_{x} \zeta^{s}+c^{-} \partial_{x} \dot{v}^{-}=0, \\
\partial_{t} \zeta+c^{+} \partial_{x} \zeta+H^{+} \partial_{x} \dot{v}^{+}=0, \\
\partial_{t} \dot{v}+g^{\prime} \nabla \zeta+\llbracket \underline{\rho}^{ \pm} c^{ \pm} \partial_{x} \dot{v}^{ \pm} \rrbracket=0,
\end{array}\right.
$$

where as usual $\dot{v}=\rho^{+} \dot{v}^{+}-\rho^{-} \dot{v}^{-}$. Proceeding as for Lemma 3 to derive a free surface version of Lemma 2 , one readily obtains that the free surface version of the linearized SW/SW system (32) takes the following form,

$$
\partial_{t} W+A_{S W}(D) W=0
$$

where $W=\left(\zeta^{s}, \dot{v}^{-}, \zeta, \dot{v}\right)$ with the notation

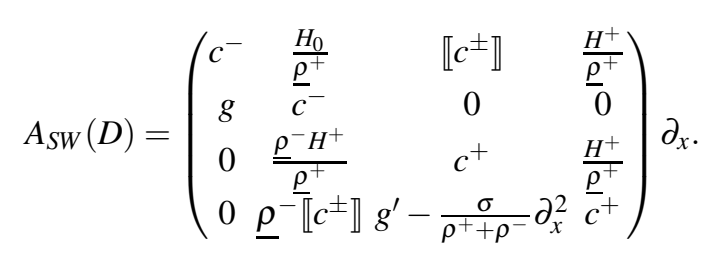

\subsubsection{The free surface SW/SW model and the Kelvin-Helmholtz instabilities}

Similar as for the full system case, the stability criterion for (46) is equivalent to the fact that the Fourier mode $A_{S W}(k)$ has purely imaginary eigenvalues for all $k$, which leads to the following condition 
Fig. 7 Stable areas of the free surface SW/SW model compared to the full system $(\sigma=0)$. Parameters $H^{+}=$ $0.62, \delta=0.15 / 0.62, \gamma=0.4$ and $g=9.81$.

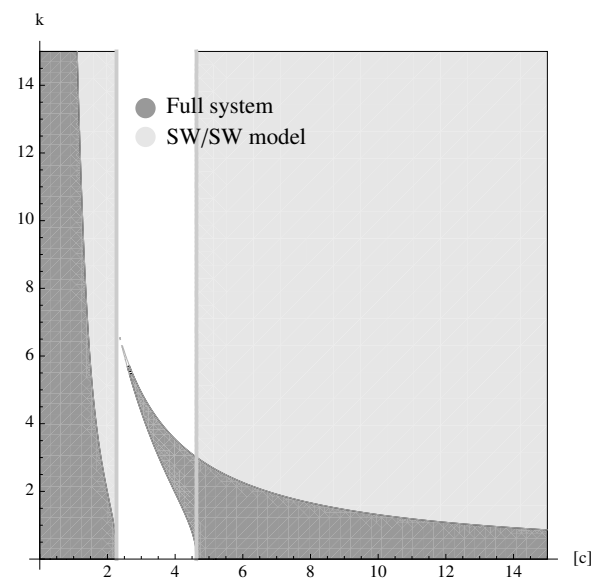

$$
\begin{gathered}
x^{4}+2 \llbracket c^{ \pm} \rrbracket x^{3}+\left(\llbracket c^{ \pm} \rrbracket^{2}-g\left(H^{+}+H^{-}\right)-\frac{\sigma H^{+}}{\underline{\rho}^{+}\left(\rho^{+}+\rho^{-}\right)} k^{2}\right) x^{2}-2 g H^{-} \llbracket c^{ \pm} \rrbracket x \\
-g H^{-} \llbracket c^{ \pm} \rrbracket^{2}+g \frac{H^{+} H^{-}}{\underline{\rho}^{+}}\left(g^{\prime}+\frac{\sigma}{\rho^{+}+\rho^{-}} k^{2}\right)=0 \quad \text { has four real roots. }
\end{gathered}
$$

With the same notations as in $\S 5.2$, this equation has four real roots if and only if

$$
\Delta_{3}^{S W}>0, \Delta_{5}^{S W}>0, \Delta_{7}^{S W}>0 \quad \text { or } \quad \Delta_{3}^{S W} \geq 0, \Delta_{5}^{S W}=0, \Delta_{7}^{S W}=0 .
$$

Since the surface tension at the interface affects only the high frequency part, we omit the surface tension i.e. set $\sigma=0$ in the following computations. Direct computations show that

$$
\Delta_{3}^{S W}=4 \llbracket c^{ \pm} \rrbracket^{2}+8 g\left(H^{+}+H^{-}\right)>0
$$

and

$$
\begin{aligned}
\Delta_{5}^{S W}=8 g & {\left[\left(H^{+}+H^{-}\right)\left(\llbracket c^{ \pm} \rrbracket^{2}-g\left(H^{+}-H^{-}\right)\right)^{2}+4 \llbracket c^{ \pm} \rrbracket^{2} g\left(3 H^{+}-H^{-}\right) H^{-}\right] } \\
& +16 g^{2} \underline{\rho}^{-} H^{+} H^{-}\left(\llbracket c^{ \pm} \rrbracket^{2}+2 g\left(H^{+}+H^{-}\right)\right) / \underline{\rho}^{+}
\end{aligned}
$$

We can tell from the expression above that $\Delta_{5}^{S W}>0$ at least when $3 H^{+}>H^{-}$ (our data in figures fit the case), therefore the stability is governed by the condition $\Delta_{7}^{S W}>0$; but the sign of $\Delta_{7}^{S W}$ in general can only be assessed numerically. Figure 7 shows how the free surface SW/SW model behaves compared to the full system.

In the particular case where $H^{+}=H^{-}=H$, the expression for the inner determinants $\Delta_{5}^{S W}$ and $\Delta_{7}^{S W}$ can be simplified considerably and one finds

$$
\Delta_{5}^{S W}=16 \llbracket c^{ \pm} \rrbracket^{4} g H+16 \llbracket c^{ \pm} \rrbracket^{2}\left(4+\underline{\rho}^{-} / \underline{\rho}^{+}\right) g^{2} H^{2}+64\left(\underline{\rho}^{-} / \underline{\rho}^{+}\right) g^{3} H^{3}>0,
$$


and

$\Delta_{7}^{S W}=16 g^{2} H^{2}\left(\llbracket c^{ \pm} \rrbracket^{2}+\left(\underline{\rho}^{-} / \underline{\rho}^{+}\right) g H\right)^{2}\left(\llbracket c^{ \pm} \rrbracket^{4}-8 \llbracket c^{ \pm} \rrbracket^{2} g H+16\left(1-\underline{\rho}^{-} / \underline{\rho}^{+}\right) g^{2} H^{2}\right)$.

Therefore the stability criterion for the linear SW/SW system (46) generated from $\Delta_{7}^{S W}>0$ reads

$$
\llbracket c^{ \pm} \rrbracket^{4}-8 \llbracket c^{ \pm} \rrbracket^{2} g H+16\left(1-\underline{\rho}^{-} / \underline{\rho}^{+}\right) g^{2} H^{2}>0 .
$$

So the stability is achieved if and only if

$$
\llbracket c^{ \pm} \rrbracket^{2}<4 g H(1-\sqrt{\gamma}) \quad \text { or } \quad \llbracket c^{ \pm} \rrbracket^{2}>4 g H(1+\sqrt{\gamma}),
$$

where we recall that $\gamma$ is the density ratio: $\gamma=\rho^{-} / \rho^{+} \in[0,1)$. Remarking that the threshold $\Omega_{K H}$ obtained in the rigid lid case for the stability of low wavenumbers can be written $\Omega_{K H}=g H \frac{1-\gamma^{2}}{\gamma}$, we obtain the following condition for the stability of the free surface SW/SW model,

$$
(\text { Stab } 1)_{S W} \quad \llbracket c^{ \pm} \rrbracket^{2}<\Omega_{K H} \times 4 \gamma \frac{1-\sqrt{\gamma}}{1-\gamma^{2}} \quad \text { or } \quad \llbracket c^{ \pm} \rrbracket^{2}>\Omega_{K H} \times 4 \gamma \frac{1+\sqrt{\gamma}}{1-\gamma^{2}} ;
$$

this is consistent with [37] where it is proved the the free surface SW/SW model is hyperbolic ${ }^{5}$ if and only if $\llbracket c^{ \pm} \rrbracket<\Omega_{K H}^{-}$or $\llbracket c^{ \pm} \rrbracket>\Omega_{K H}^{+}$where $\Omega_{K H}^{ \pm}$are two non explicit constants). This is of course equivalent to the condition for the stability of low wavenumbers derived in $\S 5.2$ for the full system. As in the rigid lid case, stability of low wavenumbers for the SW/SW model is equivalent to the stability of all wavenumbers, i.e.

$$
(\operatorname{Stab} 2)_{S W} \quad \Longleftrightarrow \quad(\operatorname{Stab} 1)_{S W} .
$$

\subsection{Behavior of the Kelvin-Helmholtz instabilities in the rigid lid limit}

We have seen in the previous section that there are at least two major differences for the behavior of Kelvin-Helmholtz instabilities in the rigid lid and in the free surface cases. Namely,

(1) In both cases, the linearized equations are stable for small shears, but the (Stab 1) condition for the stability of low frequencies differ. These conditions are given by

\footnotetext{
${ }^{5}$ Hyperbolicity does not imply well posedness of the nonlinear system (45); a stronger sufficient well-posedness condition is derived in [24, 37]; contrary to (Stab 1) $)_{S W}$, this condition is satisfied only for small shears.
} 
$\llbracket c^{ \pm} \rrbracket<\Omega_{K H} \quad($ rigid lid $) \quad$ and $\quad \llbracket c^{ \pm} \rrbracket<\Omega_{K H, 1}(0) \neq \Omega_{K H} \quad$ ( free surface )

(the fact that $\Omega_{K H, 1}(0) \neq \Omega_{K H}$ is either checked numerically or, in the particular case where $H^{+}=H^{-}$, using the explicit expression computed in $\S 6.1 .3$ ).

(2) There exists in the free surface case a zone of stability for low frequency modes when the shear is large. This stability area represented in Figure 6 and also noticed in $[35,1,11,40]$ does not exist in the rigid lid case.

Since the rigid lid approximation is much simpler and often used in applications, we discuss here whether the two differences described above are compatible with the rigid lid approximation.

From (1), one can infer that the rigid lid approximation can only be correct if $\Omega_{K H, 1}(0) \rightarrow \Omega_{K H}$ in the rigid lid limit (i.e. when the amplitude of the surface perturbations is much smaller than the interface displacement). From the explicit expression of $\Omega_{K H, 1}(0)$ computed in $\S 6.1 .3$, this implies that one necessarily has $\gamma \rightarrow 1$, i.e. that the densities $\rho^{ \pm}$of both fluids become very similar (which is the case for oceanographic applications). This is in this context that the rigid lid approximation for the SW/SW model (45) has been justified in [26]; note also that it is possible to derive a simpler model in this framework by making the so called Boussinesq approximation [9].

The question raised by (2) is: what happens to the second stability area in the rigid lid limit? This question is best answered by working in dimensionless variables

$$
\tilde{X}=\frac{X}{L}, \quad \tilde{\zeta}^{s}=\alpha \frac{\zeta^{s}}{H_{0}}, \quad \tilde{\zeta}=\frac{\zeta}{H_{0}}, \quad \tilde{v}=\frac{v}{v_{0}}, \quad \tilde{t}=t \frac{v_{0}}{L}
$$

where $v$ stand here for all the velocities involved in the equations $\left(v=v^{ \pm}, v^{s}, c^{ \pm}, \ldots\right)$, $L$ is the typical horizontal length, $H_{0}=\underline{\rho}^{+} H^{-}+\underline{\rho}^{-} H^{+}$, and $v_{0}$ is the typical speed of the linearized equations in shallow water and in the rigid lid case, $v_{0}^{2}=g^{\prime} H_{0}$ (and the rigid lid limit corresponds to $\alpha \rightarrow 0$ ). In these dimensionless variables, the (Stab $1)_{S W}$ condition for the free surface SW/SW model derived in $\S 6.1 .3$ is written

$$
(\text { Stab } 1)_{S W} \quad \llbracket \tilde{c}^{ \pm} \rrbracket^{2}<4(1+\gamma)^{2} \frac{1-\sqrt{\gamma}}{1-\gamma^{2}} \quad \text { or } \llbracket \llbracket \tilde{c}^{ \pm} \rrbracket^{2}>4(1+\gamma)^{2} \frac{1+\sqrt{\gamma}}{1-\gamma^{2}}
$$

with $\llbracket \tilde{c}^{ \pm} \rrbracket=\llbracket c^{ \pm} \rrbracket / \sqrt{g^{\prime} H_{0}}$.

As seen above, the rigid lid limit can only be relevant if $\varepsilon:=1-\gamma$ goes to zero. The above stability criterion is therefore asymptotically equivalent to

$$
(\text { Stab } 1)_{S W} \quad \llbracket \tilde{c}^{ \pm} \rrbracket^{2}<4 \text { or } \llbracket \llbracket \tilde{c}^{ \pm} \rrbracket^{2}>\frac{16}{\varepsilon} .
$$

In the rigid lid limit, the behavior of the two stability areas observed in the free surface case is the following: the left part (small shear) converges to the stability area of the rid lid model, while the right part (large shear) is shifted more to the right (see Figure 8). In the rigid lid limit, the large shear stability condition is therefore trivial in the sense that it requires an infinite shear to be satisfied. 


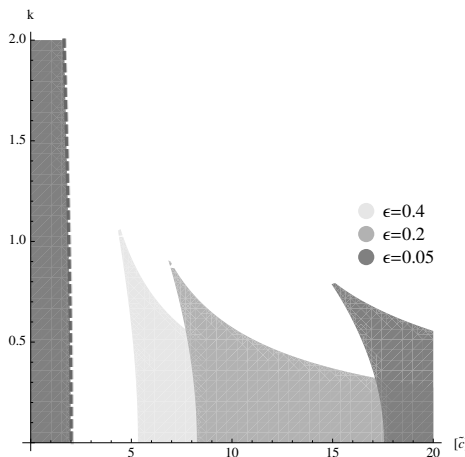

(a) the stable areas with different $\varepsilon$.

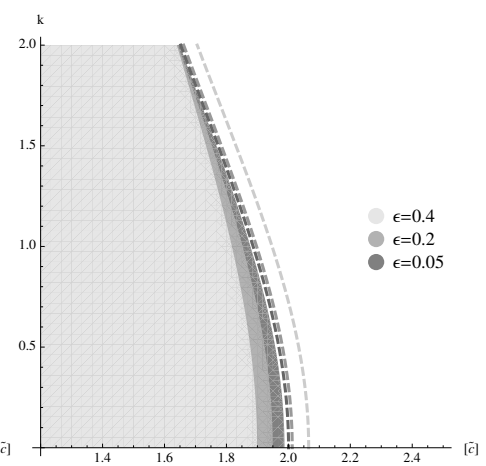

(b) a closer look at the left part.

Fig. 8 The stable areas for the linear system (43) with different parameter $\gamma=1-\varepsilon$. The horizontal axis is rescaled to be $\llbracket \tilde{c}^{ \pm} \rrbracket$ (written as $[\tilde{c}]$ in the figures ) in order to compare with the rigid lid case. The dashed lines represent the bounds for the stable area in the corresponding rigid lid cases. Parameters $\rho^{+}+\rho^{-}=2021, H^{+}=0.62=H^{-}, \sigma=0.073$ and $g=9.81$.

\subsection{Remarks on other interesting limits}

\subsubsection{The one-fluid limit $\gamma \rightarrow 0$}

It is proved in [32] that, in the rigid-lid case, the full (nonlinear) two-fluids equations converge to the one-fluid (water waves) equation as $\gamma \rightarrow 0$ (it is in particular shown that it is possible to consider simultaneously $\gamma \rightarrow 0$ and $\mathrm{Bo} \rightarrow \infty$ and to get convergence to the water waves equations without surface tension).

It is natural to wonder what happens in the free surface case for which it is expected that the stability region grows larger as $\gamma \rightarrow 0$ since for the linearized water waves equations, all wave numbers are stable (there is no Kelvin-Helmholtz instability). As shown in Fig. 9, we numerically get that when $\gamma \rightarrow 0$, the left and the right parts of the stable area in the free surface case become closer and closer and get united as one piece in the limit. For the small numbers, this phenomenon becomes transparent by looking into the condition (Stab 1$)_{S W}$ and setting $\gamma \rightarrow 0$.

\subsubsection{The small aspect ratio limit $\delta \rightarrow 0$}

To different configurations lead to a small aspect ratio $\delta=H^{-} / H^{+}$:

- When $\mathrm{H}^{+}$is fixed and $\mathrm{H}^{-}$gets smaller and smaller. In this case, the one fluid case is expected to be recovered. Consequently, the stable area in the corresponding two-fluids case with free surface should grow to cover the whole range of wavenumbers. This scenario is numerically confirmed, as shown in Fig. 10. The right 


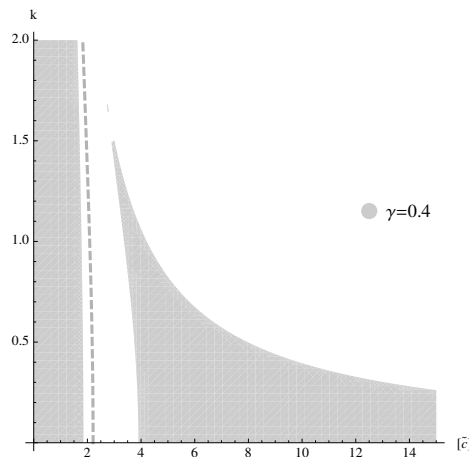

(a) Stable area when $\gamma=0.4$

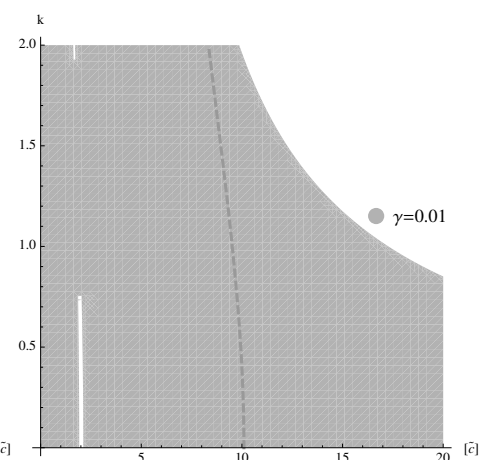

(b) Stable area when $\gamma=0.01$.

Fig. 9 The stable areas for the linear system (43) with parameter $\gamma \rightarrow 0$. The dashed lines represent the bounds for the stable area in the corresponding rigid lid cases. Parameters $\rho^{+}+\rho^{-}=2021$, $H^{+}=0.62=H^{-}, \sigma=0.073$ and $g=9.81$.

part of the stable area gets larger and larger. Meanwhile, the left part becomes closer and closer to the stable area in the rigid lid case.

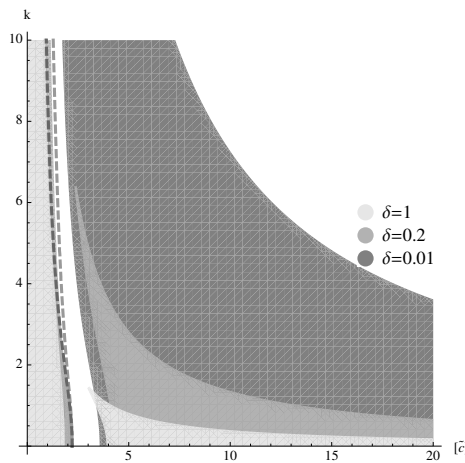

(a) the stable areas with different $\delta$.

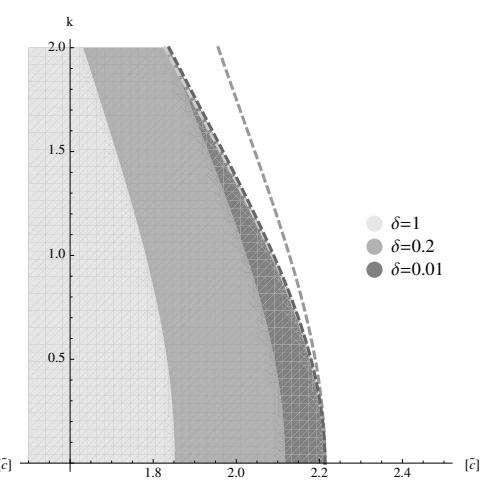

(b) a closer look at the left part.

Fig. 10 The stable areas for the linear system (43) with different parameter $\delta$ where $H_{+}=0.62$ kept fixed. The dashed lines represent the bounds for the stable area in the corresponding rigid lid cases. Parameters $\rho^{+}+\rho^{-}=2021, \gamma=0.4, \sigma=0.073$ and $g=9.81$.

- When $H^{-}$is kept fixed and $H^{+}$grows larger and larger. In this case, the rigid lid limit is expected to be valid, and it is interesting to look at the behavior of the high frequency stable area specific to the free surface case. This behavior is represented in Fig. 11. 
Fig. 11 The stable areas for the linear system (43) with different parameter $\delta$ where $H_{-}=0.15$ kept fixed. The dashed lines represent the bounds for the stable area in the corresponding rigid lid cases again. Parameters $\rho^{+}+\rho^{-}=2021, \gamma=0.4$, $\sigma=0.073$ and $g=9.81$.

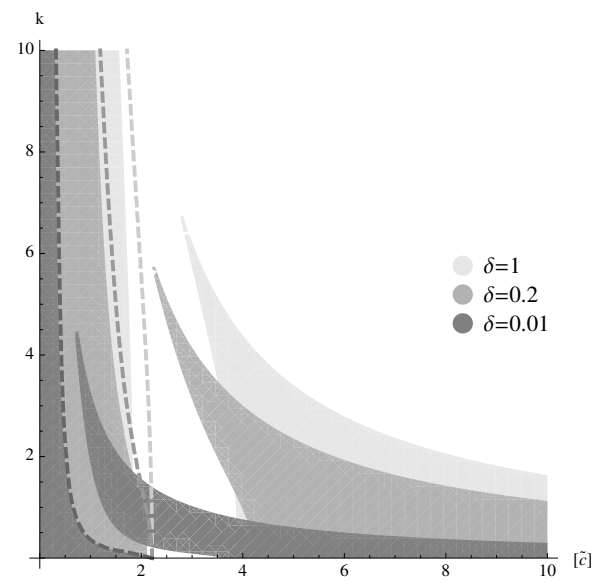

Acknowledgements The authors want to address their warmest thanks to the referee for his/her careful reading and valuable suggestions.

D. L. acknowledges support from the ANR-13-BS01-0003-01 DYFICOLTI and the ANR BOND. M.. This work was supported by Fondation Sciences Mathématiques de Paris (FSMP) when M. Ming was a postdoc with D. L. at DMA, l'École Normale Supérieure in 2012.

\section{References}

1. Barros, R., and Choi, W.: Inhibiting Shear Instability Induced by Large Amplitude Internal Solitary Waves in Two-Layer Flows with a Free Surface. Studies in Applied Mathematics 122, 325-346 (2009)

2. Barros, R., and Choi, W.: On regularizing the strongly nonlinear model for two-dimensional internal waves. Physica D 264, 27-34 (2013)

3. Barros, R., Gavrilyuk, S., and Teshukov, V. M.: Dispersive nonlinear waves in two-layer flows with free surface. I. Model derivation and general properties. Stud. Appl. Math. 119, 191-211 (2007)

4. Benjamin, T. B., and Bridges, T. J.: Reappraisal of the Kelvin-Helmholtz problem. II. Interaction of the Kelvin-Helmholtz, superharmonic and Benjamin-Feir instabilities. J. Fluid Mech. 333, 327-373 (1997)

5. Bona, J. L., Chen, M., and Saut, J. -C.: Boussinesq equations and other systems for smallamplitude long waves in nonlinear dispersive media. I. Derivation and linear theory. J. Nonlinear Sci. 12, 283-318 (2002)

6. Bona, J. L., Chen, M., and Saut, J. -C.: Boussinesq equations and other systems for small amplitude long waves in nonlinear dispersive media. II. The nonlinear theory. Nonlinearity 17, 925-952 (2004)

7. Bona, J. L., Colin, T., and Lannes, D.: Long wave approximations for water waves. Arch. Ration. Mech. Anal. 178, 373-410 (2005)

8. Bona, J. L., Lannes, D., and Saut, J. -C.: Asymptotic models for internal waves. J. Math. Pures Appl. 89, 538-566 (2008)

9. Boonkasame, A., and Milewski, P. A.: The stability of large-amplitude shallow interfacial non-Boussinesq flows, to appear in Studies in Applied Math 
10. Bresch, D., and Renardy, M.: Well-posedness of two-layer shallow-water flow between two horizontal rigid plates. Nonlinearity 24, 1081-1088 (2011)

11. Bresch, D., and Renardy, M.: Kelvin-Helmholtz instability with a free surface, Z. Angew. Math. Phys. 64, 905-915 (2013)

12. Castro, A., and Lannes, D.: Well-posedness and shallow-water stability for a new Hamiltonian formulation of the water waves equations with vorticity, submitted, http://arxiv.org/abs/1402.0464

13. Choi, W., Barros, R., and Jo, T. -C.: A regularized model for strongly nonlinear internal solitary waves. J. Fluid Mech. 629, 73-85 (2009)

14. Choi, W., and Camassa, R.: Weakly nonlinear internal waves in a two-fluid system. J. Fluid Mech. 313, 83-103 (1996)

15. Choi, W., and Camassa, R.: Fully nonlinear internal waves in a two-fluid system. J. Fluid Mech. 396, 1-36 (1999)

16. Cotter, C. J., Holm, D. D., and Percival, J. R.: The square root depth wave equations. Proc. R. Soc. Lond. Ser. A Math. Phys. Eng. Sci. 466, 3621-3633 (2010)

17. Craig, W.: An existence theory for water waves and the Boussinesq and Korteweg-de Vries scaling limits. Commun. Partial Differ. Equations 10, 787-1003 (1985)

18. Craig, W., Guyenne, P., and Kalisch, H.: A new model for large amplitude long internal waves. C. R. Mecanique 332, 525-530 (2004)

19. Craig, W., Guyenne, P., and Kalisch, H.: Hamiltonian long-wave expansions for free surfaces and interfaces. Commun. Pure Appl. Math. 58, 1587-1641 (2005)

20. Craig, W., Guyenne, P., and Sulem, C.: Coupling between internal and surface waves. Nat. Hazards 57, 617-642 (2010)

21. Craig, W., Guyenne, P., and Sulem, C.: The surface signature of internal waves. J. Fluid Mech. 710, 277-303 (2012)

22. Craig, W., and Sulem, C.: Numerical simulation of gravity waves. J. Comput. Phys 108, 73-83 (1993)

23. Drazin, P. G., and Reid, W. H.: Hydrodynamic stability. Cambridge Mono- graphs on Mechanics and Applied Mathematics. Cambridge University Press, Cambridge-New York (1982)

24. Duchêne, V.: Asymptotic shallow water models for internal waves in a two-fluid system with a free surface. SIAM Journal on Mathematical Analysis 42, 2229-2260 (2010)

25. Duchêne, V.: Asymptotic models for the generation of internal waves by a moving ship, and the dead-water phenomenon. Nonlinearity 24, 2281-2323 (2011)

26. Duchêne, V.: On the Rigid-Lid Approximation for Two Shallow Layers of Immiscible Fluids with Small Density Contrast. J. Nonlinear Sci. 24, 579-632 (2014)

27. Duchêne, V., Israwi, S., and Talhouk, R.: Shallow water asymptotic models for the propagation of internal waves. Discrete Contin. Dyn. Syst. Ser. S 7 (2014)

28. Fuller, A. T.: Root location criteria for quartic equations. IEEE Trans. Automat. Contr. AC-26, 777-782 (1981)

29. Grue, J., Jensen, A., Rusås, P. -O., and Sveen, J. K.: Properties of large-amplitude internal waves. J. Fluid Mech. 380, 257-278 (1999)

30. Guyenne, P., Lannes, D., and Saut, J. -C.: Well-posedness of the Cauchy problem for models of large amplitude internal waves. Nonlinearity 23, 237-275 (2010)

31. Lannes, D.: Well-posedness of the water-waves equations. J. Amer. Math. Soc. 18, 605-654 (2005)

32. Lannes, D.: A stability criterion for two-fluid interfaces and applications. Arch. Ration. Mech. Anal. 208, 481-567 (2013)

33. Lannes, D.: The Water Waves Problems. Mathematical Analysis and Asymptotics. Mathematical Surveys and Monographs 188. AMS (2013) 
34. Lannes, D., and Bonneton, P.: Derivation of asymptotic two-dimensional time-dependent equations for surface water wave propagation. Phys. Fluids 21, 016601 (2009)

35. Liska, R., Margolin, L., and Wendroff, B.: Nonhydrostatic two-layer models of incompressible flow. Comput. Math. Appl. 29, 25-37 (1995)

36. Miyata, M.: Long internal waves of large amplitude. In Proceedings of the IUTAM Symposium on Nonlinear Water Waves (ed. H. Horikawa \& H. Maruo), pp. 399-406. SpringerVerlag, Berlin (1988)

37. Monjarret, R.: Local well-posedness of the two-layer shallow water model with free surface, submitted.

38. Nguyen, H. Y., and Dias, F.: A boussinesq system for two-way propagation of interfacial waves.Phys. D 237, 2365-2389 (2008)

39. Nwogu, O.: Alternative form of boussinesq equations for nearshore wave propagation. J. Wtrwy., Port, Coast., and Oc. Engrg. 119, 616-638 (1993)

40. Stewart, A. L., and Dellar, P. J.: Multilayer shallow water equations with complete coriolis force. part 3. hyperbolicity and stability under shear. Journal of Fluid Mechanics 723, 289-317 (2013)

41. Whitham, G. B.: Linear and nonlinear waves. Pure and Applied Mathematics (Reprint of the 1974 original, A Wiley-Interscience Publication). John Wiley \& Sons Inc., New York (1999)

42. Wu, S.: Well-posedness in sobolev spaces of the full water wave problem in 3-d. J. Amer. Math. Soc. 12, 445-495 (1999)

43. Zakharov, V. E.: Stability of periodic waves of finite amplitude on the surface of a deep fluid. Journal of Applied Mechanics and Technical Physics 9, 190-194 (1968) 